\title{
Dynamics of Unilateral and Bilateral Control Systems with State Feedback for Renewable Resource Management
}

\author{
Mingzhan Huang $\mathbb{D}^{1},{ }^{1}$ Shouzong Liu $\mathbb{D}^{1},{ }^{1}$ Xinyu Song $\mathbb{D}^{1},{ }^{1}$ and Lansun Chen $\mathbb{D}^{2}$ \\ ${ }^{1}$ College of Mathematics and Statistics, Xinyang Normal University, Xinyang 464000, China \\ ${ }^{2}$ Institute of Mathematics, Academy of Mathematics and System Sciences, Academia Sinica, Beijing 100080, China \\ Correspondence should be addressed to Xinyu Song; xysong88@163.com
}

Received 24 November 2019; Accepted 4 February 2020; Published 10 March 2020

Academic Editor: Thierry Floquet

Copyright (c) 2020 Mingzhan Huang et al. This is an open access article distributed under the Creative Commons Attribution License, which permits unrestricted use, distribution, and reproduction in any medium, provided the original work is properly cited.

\begin{abstract}
In this paper, mathematical models for the management of biological resources based on a given predator-prey relationship are proposed, and two types of control strategies, unilateral and bilateral control with impulsive state feedback, are studied. The existence of the order-1 homoclinic orbit, order-1 periodic solution, and bifurcation of homoclinic of the unilateral control system are obtained, and the attraction region of this system is also discussed. Besides, sufficient conditions for the existence and stability of the order-1 and order-2 periodic solutions of the bilateral control system are also gained. A series of numerical simulations including bifurcation diagrams of periodic solution are performed, which not only verify the theoretical results we get but also reveal some peculiar dynamical phenomena, such as the appearance of high-order periodic solutions and existence of parameter intervals with drastic order change of periodic solution. By comparing the two management strategies, our study encourages bilateral control rather than unilateral control for the risk of predator extinction.
\end{abstract}

\section{Introduction}

Reasonable exploitation of renewable resources has been a topic drawing close attention of researchers, resource owners, and resource management departments [1]. Enormous contributions have been made on sustainable management and exploitation of renewable resources [1-10]. However, the previous studies focused more on the fluctuating environment and different policies. Srinivasu et al. proposed optimization models for resource management and investigated the impact of periodic varying environment and harvest policies on the revenue [1]. Emilie Lindkvist et al. presented a new method to investigate the influence mechanism of several different factors to the management strategies of renewable resources by applying a computational form of adaptive management [11]. Stochastic factors and resource-constrained control tasks were also considered in the management process [12-14].

A dynamic model for renewable resource management has played an important role in promoting the sustainable development of a region or a country due to its ability to anticipate future trend. In order to design management strategies of sustainable development, a variety of mathematical models have been constructed to study the impacts of different significant influence factors. Clark studied mathematical models of commercial fishing taking into consideration the economic and environmental factors [2]. Then with some investigations, Leung and Wang [15] presented a commercial fishing model incorporating capital investment. Ragozin and Brown [16] studied a model for a predator-prey system where the prey is supposed to have no commercial value while the predator can benefit humans. In contrast, Mesterton-Gibbons investigated a predator-prey system where the two species are both harvested with an optimal policy [17]. A nonautonomous model with periodic coefficients was proposed by Fan and Wang in [18], and they studied the positive periodic solution and its stability.

Feedback control was also used in the general renewable resource management. Sandal and Steinshamn in [19] studied the optimal exploitation of renewable natural resources by a feedback model. They presented some feedback 
rules in computing optimal yields and depletion rates by applying perturbation theory. They also found the analytical expressions of the optimal harvest under an explicit feedback control law [12]. Fuertes et al. gave an optimal resource allocation policy aiming at maximizing the control performance and also provided experimental comparisons to show the priority of this policy [14].

With the rapid development of theories and methods of the semicontinuous dynamic system in recent years, a large number of mathematical models of renewable resource management considering state feedback control are formulated and studied. Xiao and Dai studied a predator-prey system with Allee effect and state feedback and discussed the coexistence mode of the two biological resources [20]. Huang et al. constructed a population system with two types of harvesting, and they exploited the impact of the harvest effort on the development trend of population [21]. Liang et al. formulated a predator-prey system with mutual interference and also investigated the dynamical behaviors of the system [22]. Besides, Chen and Zhao proposed a delayed singlespecies model with impulsive control, and they mainly discussed the existence and stability of order-1 periodic solutions [23]. Furthermore, Guo et al. in [24] investigated a state feedback for the algae-fish system while Fu and Chen in [25] formulated an ecological system for water hyacinth and studied two types of state-dependent impulsive controls.

Overexploitation or excessive use of a renewable resource that has economic value can be a threat to biodiversity. As we all know, all species do not exist in isolation, and they will interact with other species around them. A species may be the prey, or the predator, or a competitor of another species. So when it is overexploited, what will happen to the other one? And what should humans do to protect the biodiversity of the ecological system? Based on these considerations, in this paper by constructing semicontinuous dynamic systems, we present mathematical models for the management of two kinds of biological resources based on a given predator-prey relationship. Since the quantity of the prey population can be easily obtained by some modern devices and measurement techniques, we design two control strategies, unilateral and bilateral control, with the state feedback of the prey population.

The paper is organized as follows. In Section 2, we formulate two mathematical models for the unilateral and bilateral state feedback control of the two kinds of biological resources. In Section 3, we give qualitative analysis for the unilateral and bilateral control systems, and dynamical behaviors such as the existence and stability of order- 1 and order-2 periodic solutions and bifurcation phenomenon corresponding to control parameters are investigated. In Section 4, series of numerical simulations including bifurcation diagrams of periodic solution are performed, which not only verify the theoretical results we get but also reveal some peculiar dynamical phenomena. We finish this paper with a brief discussion in Section 5 .

\section{Model Formulation}

2.1. Basic Model. The basic model studied in this paper is the following predation system [26]:

$$
\left\{\begin{array}{l}
\frac{\mathrm{d} x(t)}{\mathrm{d} t}=r x(t)\left(1-\frac{x(t)}{K}\right)-\alpha x(t) y(t) e^{-\beta x(t)}, \\
\frac{\mathrm{d} y(t)}{\mathrm{d} t}=y(t)\left[\mu \alpha x(t) e^{-\beta x(t)}-D\right],
\end{array}\right.
$$

where $x(t)$ and $y(t)$ represent the densities of the prey and predator, respectively. $r, K, \alpha, \beta, \mu$, and $D$ are the positive constants. $\alpha x(t) e^{-\beta x(t)}$ denotes the functional response when the predation confronts group defense from the prey species.

For model (1), there are always an unstable saddle point $O(0,0)$ and a boundary equilibrium $E_{0}(K, 0)$. To get positive equilibria, we need to solve the following algebra equations:

$$
\left\{\begin{array}{l}
r\left(1-\frac{x}{K}\right)-\alpha y e^{-\beta x}=0, \\
\mu \alpha x e^{-\beta x}-D=0 .
\end{array}\right.
$$

By simple calculation, we have the following lemma.

\section{Lemma 1}

(i) If $\alpha \mu<e \beta D$, then system (1) has no positive equilibrium;

(ii) if $\alpha \mu=e \beta D$ and $(1 / \beta)<K$, then system (1) has a unique positive steady state $((1 / \beta),(\mathrm{re} / \alpha)(1-$ $(1 / K \beta)))$;

(iii) if $\alpha \mu>e \beta D$, then system (1) has at most two positive equilibria $E_{1}\left(x_{1}, y_{1}\right)$ and $E_{2}\left(x_{2}, y_{2}\right)$, where $x_{i}, i=1,2$ satisfy $\mu \alpha x_{i} e^{-\beta x_{i}}=D$ and $0<x_{1}<(1 / \beta)<x_{2}<K$.

For case (iii) in Lemma 1, Xiao and Ruan in [26] had discussed two cases: when system (1) has a global asymptotically stable equilibrium $E_{1}\left(x_{1}, y_{1}\right)$ and when it has an unstable equilibrium $E_{1}\left(x_{1}, y_{1}\right)$ and a saddle $E_{2}\left(x_{2}, y_{2}\right)$ (interested readers can refer Theorems 1 and 2 in [26]).

Now, we give other conditions under which the existence of the two interior equilibria is guaranteed, and $E_{1}\left(x_{1}, y_{1}\right)$ is stable while $E_{2}\left(x_{2}, y_{2}\right)$ is still a saddle.

Theorem 1. If $\alpha \mu>e \beta D$ and $x_{2}<K<(1 / \beta)+x_{1}$, then system (1) has four equilibria: two unstable saddles $O(0,0)$ and $E_{2}\left(x_{2}, y_{2}\right)$, a stable node $E_{0}(K, 0)$, and a stable node or focus $E_{1}\left(x_{1}, y_{1}\right)$.

Proof. From Lemma 1, we know that if $\alpha \mu>e \beta D$ and $x_{2}<$ $K$, the system (1) has two positive steady states $E_{1}\left(x_{1}, y_{1}\right)$ and $E_{2}\left(x_{2}, y_{2}\right)$ besides two boundary equilibria $O(0,0)$ and $E_{0}(K, 0)$. To study the stability of these equilibria, we give the Jacobi matrix of the linearized system of (1) in the following:

$$
J=\left(\begin{array}{cc}
r-\frac{2 r}{K} x-\alpha y e^{-\beta x}(1-\beta x) & -\alpha x e^{-\beta x} \\
\alpha \mu y e^{-\beta x}(1-\beta x) & \alpha \mu x e^{-\beta x}-D
\end{array}\right) .
$$


By simple calculation, we have

$$
\begin{aligned}
& J(O)=\left(\begin{array}{cc}
r & 0 \\
0 & -D
\end{array}\right), \\
& J\left(E_{0}\right)=\left(\begin{array}{cc}
-r & -\alpha K e^{-\beta K} \\
0 & \alpha K \mu e^{-\beta K}-D
\end{array}\right) \text {, } \\
& J\left(E_{i}\right)=\left(\begin{array}{cc}
\frac{\beta r x_{i}}{K}\left(K-x_{i}-\frac{1}{\beta}\right) & -\alpha x_{i} e^{-\beta x_{i}} \\
\alpha \mu y_{i} e^{-\beta x_{i}}\left(1-\beta x_{i}\right) & 0
\end{array}\right) .
\end{aligned}
$$

Obviously, $O(0,0)$ is an unstable saddle and $E_{0}(K, 0)$ is a stable node if $x_{2}<K$. At points $E_{i}\left(x_{i}, y_{i}\right), i=1,2$, the characteristic equation has the form

$$
\lambda^{2}-a_{1} \lambda+a_{2}=0
$$

where $\quad a_{1}=\left(\left(\beta r x_{i}\right) / K\right)\left(K-x_{i}-(1 / \beta)\right)$ and $a_{2}=\alpha D y_{i}$ $e^{-\beta x_{i}}\left(1-\beta x_{i}\right)$.

Because $x_{1}<(1 / \beta)<x_{2}<K<(1 / \beta)+x_{1}$, we can get $a_{2}<$ 0 when $x_{i}=x_{2}$ and $a_{2}>0$ when $x_{i}=x_{1}$; then, $E_{2}\left(x_{2}, y_{2}\right)$ is a saddle point. Besides, $x_{i}=x_{1}$ implies $a_{1}<0$, so $E_{1}\left(x_{1}, y_{1}\right)$ is a stable node or focus. The proof is completed.

Theorem 2. If $r(\beta-(1 / K))+\alpha \mu<2 \sqrt{((\beta r D) / K)}$, then there are no closed orbits for system (1) provided that the conditions listed in Theorem 1 are satisfied.

Proof. In consideration of the unstable saddle $E_{2}\left(x_{2}, y_{2}\right)$, if there is a close orbit (a periodic orbit or a homoclinic loop) of system (1), it must be in the following domain:

$$
\Omega_{1}=\left\{(x, y) \mid 0<x \leq x_{2}, 0<y<\infty\right\} .
$$

Let $\mathrm{d} t=e^{\beta x} \mathrm{~d} \tau$, then system (1) is changed into an equivalent form:

$$
\left\{\begin{array}{l}
\frac{\mathrm{d} x}{\mathrm{~d} \tau}=r x\left(1-\left(\frac{x}{K}\right)\right) e^{\beta x}-\alpha x y=P(x, y), \\
\frac{\mathrm{d} y}{\mathrm{~d} \tau}=y\left[\mu \alpha x-D e^{\beta x}\right]=Q(x, y) .
\end{array}\right.
$$

Applying Dulac function $B(x, y)=x^{-1}$, we have

$$
\frac{\partial P B}{\partial x}+\frac{\partial Q B}{\partial y}=\alpha \mu+e^{\beta x}\left[-\frac{\beta r}{K} x+r\left(\beta-\frac{1}{K}\right)-\frac{D}{x}\right] .
$$

Note that $-(\beta r / K) x-(D / x) \leq-2 \sqrt{((\beta r D) / K)}$, then we can get

$$
\frac{\partial P B}{\partial x}+\frac{\partial Q B}{\partial y} \leq \alpha \mu+e^{\beta x}\left[r\left(\beta-\frac{1}{K}\right)-2 \sqrt{\frac{\beta r D}{K}}\right] .
$$

In addition, we know that $e^{\beta x} \geq 1, x \in\left(0, x_{2}\right]$, and if $r(\beta-(1 / K))+\alpha \mu<2 \sqrt{((\beta r D) / K)}$, there is

$$
\frac{\partial P B}{\partial x}+\frac{\partial Q B}{\partial y} \leq \alpha \mu+r\left(\beta-\frac{1}{K}\right)-2 \sqrt{\frac{\beta r D}{K}}<0 .
$$

By Dulac theory, system (1) does not have a closed orbit in $\Omega_{1}$. This completes the proof.

2.2. Model Formulation. Li et al. [27] investigated impulsive control tactics of system (1) at periodic fixed moments, gained the existence and local stability of the prey-extinction periodic solution, and also exploited several kinds of bifurcation phenomena. He [28] studied system (1) incorporating impulsive state feedback controls. However, the study just focused on a special case, that is to say, system (1) has a unique positive equilibrium $E_{1}\left(x_{1}, y_{1}\right)$ which is globally stable.

In this work, we study system (1) under the conditions provided in Theorems 1 and 2; that is to say, system (1) has two positive equilibria: a hyperbolic saddle $E_{2}\left(x_{2}, y_{2}\right)$ and a stable node or focus $E_{1}\left(x_{1}, y_{1}\right)$ (see Figure 1 ).

Assume that the prey and predator are two kinds of biological resources which have economic value, while the quantity of the prey population can be easily monitored by some modern devices and measurement techniques. Our research object is providing scientific strategies for the sustainable development and reasonable utilization of these two kinds of resources.

From Figure 1, we can see that if the prey density is less than $x_{L}\left(x_{L}\right.$ is the distance between the stable manifold of $E_{2}\left(x_{2}, y_{2}\right)$ and $y$-axis) or greater than $x_{2}$, there is a high probability that the predator species goes extinct. So in the following, for the biodiversity of this ecological system, we choose a lower control threshold $h_{1}>x_{1}>x_{L}$ and an upper control threshold $h_{2}<x_{2}$ for the prey density. To avoid overexploitation of the prey population, we first choose $h_{1}$ as the control threshold. With the state feedback, once the density of the prey drops down to $h_{1}$, then people release a certain amount of the prey and harvest the predator by a specific ratio at the same time. Then, we formulate a model for the unilateral control with state feedback of the prey population:

$$
\left\{\begin{array}{l}
\frac{\mathrm{d} x(t)}{\mathrm{d} t}=r x(t)\left(1-\frac{x(t)}{K}\right)-\alpha x(t) y(t) e^{-\beta x(t)}, \\
\frac{d y(t)}{d t}=\mu \alpha x(t) y(t) e^{-\beta x(t)}-D y(t), \\
x\left(t^{+}\right)=x(t)+m_{1}, \\
y\left(t^{+}\right)=(1-p) y(t),
\end{array}\right\} x(t)=h_{1}, y>r h_{1}\left(1-\frac{h_{1}}{K}\right) e^{-\beta h_{1}},
$$

where $x(0)>x_{L}, y(0)>0, x_{1}<h_{1}<h_{1}+m_{1}<x_{2}$, and $0<p<1$.

Besides, for any prey size $x=n, x_{1}<n<x_{2}$, the predator species itself has a sustainable range $\left[n_{m i}, n_{m x}\right]$. If the predator resource is overexploited, for example, if its density is less than $n_{m i}$, then the prey size will exceed $x_{2}$ and 


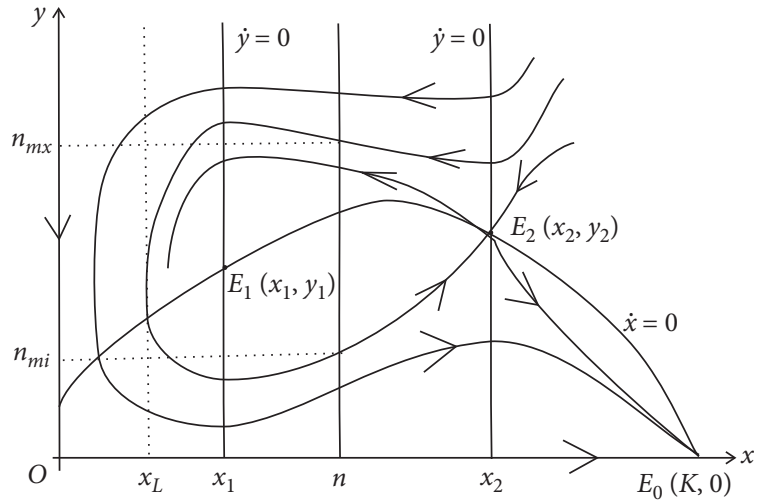

Figure 1: Phase portrait of system (1) if conditions in Theorems 1 and 2 are satisfied.

the predator will become extinct. For this reason, we set another upper control threshold $h_{2}$ besides the lower one. Then, when the density of the predator is less than $\left(h_{2}\right)_{m i}$ and the density of the prey increases to $h_{2}$, people release a certain amount of the predator and harvest a certain amount of the prey at the same time. For this case, we formulate another management strategy with bilateral control:

$$
\left\{\begin{array}{l}
\frac{\mathrm{d} x(t)}{\mathrm{d} t}=r x(t)\left(1-\frac{x(t)}{K}\right)-\alpha x(t) y(t) e^{-\beta x(t)}, \\
\frac{\mathrm{d} y(t)}{\mathrm{d} t}=\mu \alpha x(t) y(t) e^{-\beta x(t)}-D y(t), \\
\left.\begin{array}{l}
x\left(t^{+}\right)=x(t)+m_{1}, \\
y\left(t^{+}\right)=(1-p)(t),
\end{array}\right\} x(t)=h_{1}, y>r h_{1}\left(1-\frac{h_{1}}{K}\right) e^{-\beta h_{1},} \\
x\left(t^{+}\right)=x(t)-m_{2}, \\
y\left(t^{+}\right)=y(t)+\tau,
\end{array}\right\} x(t)=h_{2}, y<r h_{2}\left(1-\frac{h_{2}}{K}\right) e^{-\beta h_{2},},
$$

with initial value $h_{1}<x(0)<h_{2}, y(0)>0$. Here, $m_{1}, m_{2}, p$, and $\tau$ are the positive constants that satisfy $h_{1}+m_{1}=h_{2}-m_{2} \triangleq n$ and $x_{1}<n<x_{2}$.

The aim of this paper is to investigate the management strategies that can maintain the sustainable development of the two species with rational replenishment and exploitation by studying systems (11) and (12).

\section{Main Results}

3.1. Preliminary. Before the study of the above two systems, some notations and definitions of semidynamical systems are introduced. These will be used in the following discussion.

Consider the general planar semidynamical system:

$$
\begin{cases}\frac{\mathrm{d} x}{\mathrm{~d} t}=P(x, y), \frac{\mathrm{d} y}{\mathrm{~d} t}=Q(x, y), & (x, y) \in \mathscr{M}\{x, y\}, \\ \triangle x=\alpha(x, y), \triangle y=\beta(x, y), & (x, y) \in \mathscr{M}\{x, y\},\end{cases}
$$

where $(x, y) \in R^{2}, P(x, y), Q(x, y), \alpha(x, y)$, and $\beta(x, y) \in C$ $\left[R^{2}, R\right] . \mathscr{M} \subset R^{2}$ is called the impulsive set, while $\varphi: R^{2} \longrightarrow R^{2}$ is called the impulse mapping, where $\varphi(Z)=Z^{+}=\left(x_{Z}^{+}, y_{Z}^{+}\right)$ $=\left(x_{Z}+\alpha\left(x_{Z}, y_{Z}\right), y_{Z}+\beta\left(x_{Z}, y_{Z}\right)\right)$ for $Z\left(x_{Z}, y_{Z}\right) \in \mathscr{M} . Z^{+}$ is the phase point of point $Z$, and $\mathcal{N}=\varphi(\mathscr{M})$ is the phase set.

Assume $X(t)=(x(t), y(t))$ be an arbitrary solution of (5). Denote $\pi_{t}\left(X_{0}, X\right)=X(t)$ to be the solution corresponding to initial value condition $X(0)=X_{0}$. Then, its forward orbit can be described by $\pi\left(X_{0}, X\right)=\left\{\pi_{t}\left(X_{0}, X\right) \mid t \geq 0\right\}$, which is also denoted by $\pi(X)$ or $\pi_{+}(X)$ for simplicity. We denote $X_{k}=X\left(t_{k}^{+}\right) \in \pi(X)$, where $X\left(t_{k}\right) \in \pi(X), k=1,2, \ldots$

Definition 1 (see $[29,30]$ ). An orbit $\pi(X)$ of $(13)$ is said to be a periodic orbit if there is a $k \in N^{+}$such that $X_{k}=X_{0}$. Denote $k_{0}=\min \left\{k \in N^{+}, X_{k}=X_{0}\right\}$, then the orbit $\pi(X)$ is called an order- $k_{0}$ periodic orbit.

Definition 2 (see $[29,30])$. Assume $\pi(X)$ and $\pi(\widetilde{X})$ are the two orbits of (13), if there is a monotone smooth function $\nu(t), t>0$ such that $\left|\pi_{t}(X)-\pi_{\nu(t)}(\widehat{X})\right|\langle\epsilon, t\rangle 0$, then we called the two orbits as $\epsilon$-close.

Definition 3 (see $[29,30,31]$ ). Suppose $\pi(\widetilde{X})$ is an orbit of (13), if for any $\epsilon>0$, there must exist $\zeta>0$ such that for any point $X \in U(\tilde{X}, t \zeta), \pi(X)$ and $\pi(\widetilde{X})$ are $\epsilon$-close, then we call the orbit $\pi(\tilde{X})$ is orbitally stable; if $\pi(\tilde{X})$ is orbitally stable and there exists a $\zeta_{0}>0$ such that for all points $X \in U\left(\widetilde{X}, \zeta_{0}\right)$, we can find a constant $\delta$ such that $\left|\pi_{t}(X)-\pi_{t-\delta}(X)\right| \longrightarrow 0$, $t \longrightarrow \infty$, then we call $\pi(\widetilde{X})$ is asymptotically orbitally stable.

Lemma 2. Assume $(\xi(t), \eta(t))$ is an order- $k$ periodic solution of (13) with period T. If the Floquet multiplier $\tilde{\mu}$ satisfies $|\widetilde{\mu}|<1$, where

$$
\widetilde{\mu}=\prod_{k=1}^{n} \Delta_{k} \exp \left[\left(\int_{0}^{T} \frac{\partial P}{\partial x}(\xi(t), \eta(t))+\frac{\partial Q}{\partial y}(\xi(t), \eta(t)) \mathrm{d} t\right)\right],
$$

with

$$
\Delta_{k}=\frac{P_{+}((\partial \beta / \partial y)(\partial \varphi / \partial x)-(\partial \beta / \partial x)(\partial \varphi / \partial y)+(\partial \varphi / \partial x))+Q_{+}((\partial \alpha / \partial x)(\partial \varphi / \partial y)-(\partial \alpha / \partial y)(\partial \varphi / \partial x)+(\partial \varphi / \partial y))}{P(\partial \varphi / \partial x)+Q(\partial \varphi / \partial y)},
$$


and $P, Q,(\partial \alpha / \partial x),(\partial \alpha / \partial y),(\partial \beta / \partial x),(\partial \beta / \partial y),(\partial \varphi / \partial x)$, and $(\partial \varphi / \partial y)$ are calculated at the point $\left(\xi\left(t_{k}\right), \eta\left(t_{k}\right)\right)$, $P_{+}=P\left(\xi\left(t_{k}^{+}\right), \eta\left(t_{k}^{+}\right)\right), Q_{+}=Q\left(\xi\left(t_{k}^{+}\right), \eta\left(t_{k}^{+}\right)\right)$. Then, $(\xi(t), \eta(t))$ is orbitally asymptotically stable.

As previously mentioned, if the prey density is less than $x_{L}$ or greater than $x_{2}$, there is a high probability that the predator species goes extinct. Therefore, to get a mathematical model which is more nearing to the practice, we assume that the initial pest density is larger than $h_{1}$ and less than $x_{2}$.

In the following, we will provide a series of qualitative analysis for systems (11) and (12) in the region $\Omega$ that is surrounded by $x$-axis, the threshold line $x=h_{1}$, and the unstable flow of $E_{2}\left(x_{2}, y_{2}\right)$.

3.2. Periodic Solution, Homoclinic Bifurcation, and Attraction Region of System (11). For system (11), we denote the impulsive set by

$$
\mathscr{M}_{1}=\left\{(x, y) x=h_{1}, y>r h_{1}\left(1-\left(\frac{h_{1}}{K}\right)\right) e^{-\beta h_{1}}\right\} .
$$

while the corresponding phase set is $\mathcal{N}_{1}=\{(x, y) x=$ $\left.h_{1}+m_{1}, y>0\right\}$.

Theorem 3. If $\alpha \mu>e \beta D, x_{2}<K<(1 / \beta)+x_{1}, r(\beta-(1 / K))$ $+\alpha \mu<2 \sqrt{(\beta r} D / K)$, and $x_{1}<h_{1}<h_{1}+m_{1}<x_{2}$, then there exists two fixed parameter values $0<p^{0}<p^{*}<1$ such that

(1) If $p=p^{*}$, then system (11) admits an order-1 homoclinic cycle;

(2) If $p^{0}<p<p^{*}$, then system (11) admits a unique order1 periodic orbit in $\Omega$;

(3) If $p^{*}<p<1$, then system (11) has no periodic solution in region $\Omega$.

Proof. Let $A$ and $D$ denote the first intersection points of the unstable flow of $E_{2}\left(x_{2}, y_{2}\right)$ (in the direction of time increasing) with straight lines $x=h_{1}$ and $x=h_{1}+m_{1}$, respectively. However, point $B$ is the last intersection point of the stable flow of $E_{2}\left(x_{2}, y_{2}\right)$ (in the direction of time increasing) and the straight line $x=h_{1}+m_{1}$. Besides, lines $x=$ $h_{1}$ and $x=h_{1}+m_{1}$ intersect the isoclinic line $\dot{x}=0$ at points $C$ and $G$, respectively (see Figure 2 ). For every point $M \in \Omega$, we denote its coordinate by $M\left(x_{M}, y_{M}\right)$ and the forward orbit from it by $\pi_{+}(M)$. Because the phase set $\mathcal{N}_{1}$ is parallel to $y$ axis, for any point $S \in \mathcal{N}$, we define its coordinate as $y_{S}$ and denote the successor function of point $S$ by $f_{\text {sor }}(S)$.

Since $0<p<1$, there has two fixed parameter values $p^{0}$ and $p^{*}$ such that $\left(1-p^{0}\right) y_{A}=y_{C}$ and $\left(1-p^{*}\right) y_{A}=y_{B}$ (see the left figure in Figure 2).

(1) $p=p^{*}$. Then, there is $f_{\text {sor }}(B)=0$, and the orbit $\widehat{B E_{2}} \cup \widehat{E_{2} A} \cup \overline{A B}$ forms a homoclinic cycle, that is to say, an order-1 circle which has a saddle on it.

(2) $p^{0}<p<p^{*}$. To prove that system (11) has an order-1 periodic orbit, we need to find a point $S \in \mathcal{N}_{1}$ such that $f_{\text {sor }}(S)=0$. Assume that point $A$ jumps to point $A^{+}$due to impulsive effect; then, $A^{+}$is the successor point of $B$. Since $(1-p) y_{A}=y_{A^{+}}>y_{B}=$ $\left(1-p^{*}\right) y_{A}$, we have $f_{\text {sor }}(B)>0$. Choose point $M\left(h_{1}+m_{1}, y_{M}\right) \in \mathcal{N}_{1}$ close to point $B$ such that $y_{M}$ $>y_{B}$; then, the forward orbit $\pi_{+}(M)$ intersects $x=h_{1}$ at a point $M^{\prime}$ with $y_{A}>y_{M^{\prime}}$ and $M^{\prime}$ is mapped to point $M_{1}$ after impulsive effect. Clearly, $y_{M_{1}}>y_{M}$ and $f_{\text {sor }}(M)>0$. Furthermore, consider the orbit $\pi_{+}\left(M_{1}\right)$, we can easily get $f_{\text {sor }}\left(M_{1}\right)=y_{M_{2}}-y_{M_{1}}<0$. Since $f_{\text {sor }}$ is continuous, there must exist a point $S \in \overline{M M_{1}} \subset \mathcal{N}_{1}$ such that $f_{\text {sor }}(S)=0$ (see the right figure in Figure 2).

In the following, we discuss the uniqueness of the order-1 periodic orbit.

For any point $P\left(x_{P}, y_{P}\right) \in \mathcal{N}_{1}, y_{P}<y_{B}$, the forward orbit $\pi_{+}(P)$ tends to the stable node $E_{0}(K, 0)$. Besides, for every point $P \in \overline{C \quad D} \subset \mathcal{N}_{1}$, we have $f_{\text {sor }}(P)<0$. Therefore, if the order-1 periodic orbit exists, it must begin from $\overline{B C}$. We arbitrarily choose two points $B_{1}, B_{2} \in \overline{B C}, y_{B}<y_{B_{1}}<y_{B_{2}}<y_{C}$. Since different trajectories are disjoint, it is easy to obtain $f_{\text {sor }}\left(B_{1}\right)>$ $f_{\text {sor }}\left(B_{2}\right)$. That is to say, $f_{\text {sor }}$ is a monotonically decreasing function defined on the line segment $\overline{B C}$, and there is a unique point $S \in \overline{B C} \subset \mathcal{N}_{1}$ such that $f_{\text {sor }}(S)=0$. From the above, system (11) has a unique order-1 periodic orbit in $\Omega$ (see Figure 3 ).

(3) $p^{*}<p<1$. There is $(1-p) y_{A}<y_{B}$, so all the points $P$ on the line segment $\bar{B} \quad D \subset \mathcal{N}_{1}$ satisfy $f_{\text {sor }}(P)<0$. Besides, the forward orbit $\pi_{+}(P)$ tends to the stable node $E_{0}(K, 0)$ for any point $P\left(x_{P}, y_{P}\right) \in \mathcal{N}_{1}, y_{P}<y_{B}$. Thus, system (11) has no periodic orbit in $\Omega$. This completes the proof.

Remark 1. Choose $p$ as the bifurcation parameter. According to Theorem 3, we can get that if parameter $p$ equals to the critical value $p^{*}$, there is an order- 1 homoclinic cycle of system (11); when parameter $p$ gradually decreases from $p=p^{*}$ to $p^{0}<p<p^{*}$, the homoclinic cycle disappears while an order-1 periodic orbit appears; when parameter $p$ gradually increases from $p=p^{*}$ to $p^{*}<p<1$, the homoclinic cycle disappears but no new order-1 periodic cycle appears.

Remark 2. By Theorem 3, system (11) has an order-1 periodic orbit $\Gamma_{S}, S \in \overline{B C} \subset \mathcal{N}_{1}$. Let point $L_{0}^{+} \in U^{0}(S, \zeta) \cap \mathcal{N}_{1}$ and $\zeta>0$ is a constant that is small enough. Denote the successor point of $L_{n}^{+}$by $L_{n+1}^{+}, n=0,1,2, \ldots$. Then, there are two point sequences $\left\{L_{2 k}^{+}\right\}$and $\left\{L_{2 k+1}^{+}\right\}, k=0,1,2, \ldots$, in the phase set $\mathcal{N}_{1}$ which are separated by point $S$. Obviously, for any $n=0,1,2, \ldots$, we have $y_{L^{+}}=y_{L_{n}^{+}}+f_{\text {sor }}\left(L_{n}^{+}\right)$.

Let $d\left(S, L_{n}^{+}\right)=\left|y_{S}-y_{L^{+}}\right|$and denote $\kappa=\left(d\left(S, L_{2}^{+}\right) \mid\right.$ $\left.d\left(S, L_{0}^{+}\right)\right)$. According to Definition 3 , to prove the orbitally asymptotically stability of the order- 1 periodic orbit, we need to show that there exists at least one $\widetilde{\zeta}>0$ such that $\kappa<$ 1 for every $L_{0}^{+} \in U^{0}(\widetilde{\zeta}, S) \cap \mathcal{N}_{1}$. If there is always a point $L_{0}^{+} \in U^{0}(\zeta, S) \cap \mathcal{N}_{1}$ satisfying $\kappa>1$ for any $\zeta>0$, then we can get the unstability of the order-1 periodic orbit. If there exists a $L_{0}^{+} \in U^{0}(\zeta, S) \cap \mathcal{N}_{1}$ such that $\kappa=1$, then system (11) admits 


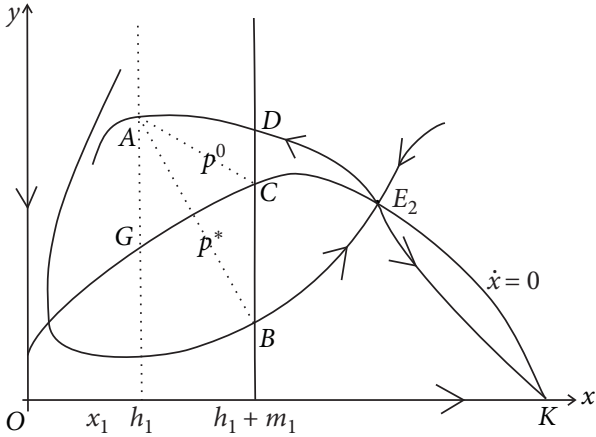

(a)

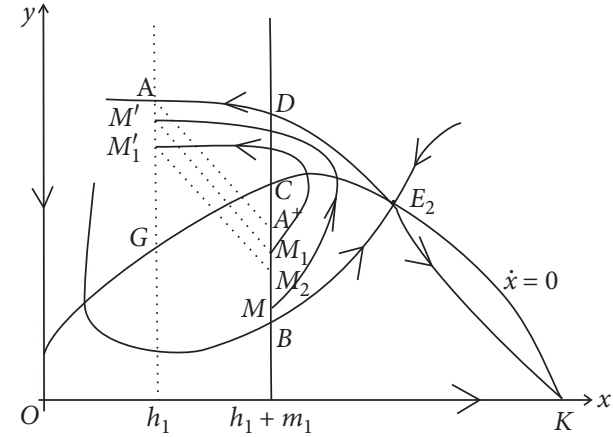

(b)

FIGURE 2: Existence of order-1 homoclinic cycle and order-1 periodic orbit of system (11).

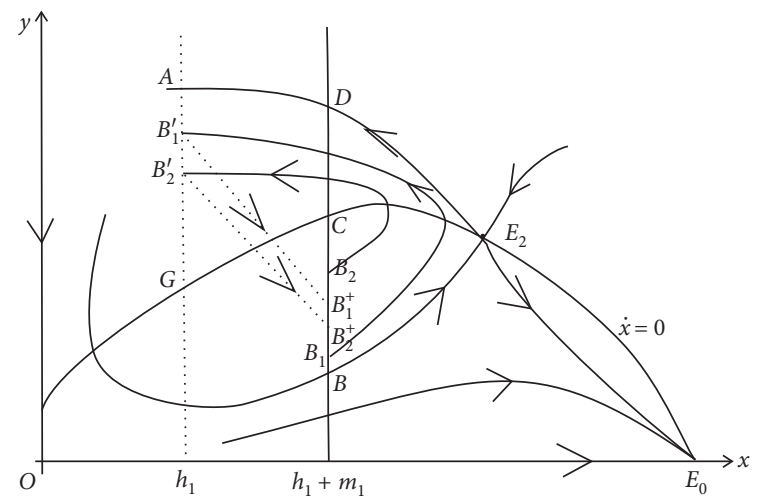

FIGURE 3: Uniqueness of order-1 periodic orbit of system (11) when $p 0<p<p^{*}$.

an order- 2 periodic orbit passing through $L_{0}^{+}$. From the analysis in Theorem 3 , we can get that function $f_{\text {sor }}$ has some good properties, for example, $f_{\text {sor }}(L)<0$ if $L \in \mathcal{N}_{1}, y_{L}>y_{S}$; $f_{\text {sor }}(L)>0$ if $L \in \mathcal{N}_{1}, y_{L}<y_{S}$; and $f_{\text {sor }}(L)=0$ if and only if $L=$ $S$. However, we cannot ensure that the order-1 periodic orbit is stable.

Theorem 4. For the case $p^{0}<p<p^{*}$ and $(1-p) y_{G}>y_{B}$, if $\alpha \mu>e \beta D, \quad x_{2}<K<(1 / \beta)+x_{1}, \quad r(\beta-(1 / K))+\alpha \mu<$ $2 \sqrt{((\beta r \quad D) / K)}$, and $x_{1}<h_{1}<h_{1}+m_{1}<x_{2}$, then system (11) has an attraction region in $\widetilde{\Omega}$, where $\widetilde{\Omega}$ is surrounded by the two separatrices of $E_{2}\left(x_{2}, y_{2}\right)$ and the threshold line $x=h_{1}$.

Proof. Let the order-1 periodic orbit $\Gamma_{S}$ intersect the impulsive set $x=h_{1}$ at point $S^{\prime}$. Denote point $B$ by point $L_{0}^{+}$. There is $f_{\text {sor }}\left(L_{0}^{+}\right)>0$ and the successor point $L_{1}^{+}$is above point $S$ (see Figure 4 ).

For $\pi_{+}\left(L_{1}^{+}\right)$, we need to determine the position of its successor point $L_{2}^{+}$. For this purpose, consider the forward orbit $\pi_{+}\left(L_{1}^{+}\right)$and it meets the impulsive set $x=h_{1}$ at point $L_{1}^{\prime}$. Obviously, $L_{1}^{\prime}$ is between two points $S^{\prime}$ and $G$. According to the conditions $p^{0}<p<p^{*}$ and $(1-p) y_{G}>y_{B}$, we get $(1-p) y_{G}<(1-p) y_{L_{1}^{\prime}}$, so the successor point of $L_{1}^{+}$ (denoted by $L_{2}^{+}$) is above the point $B$ and we have $L_{0}^{+}<L_{2}^{+}<y_{S}$.

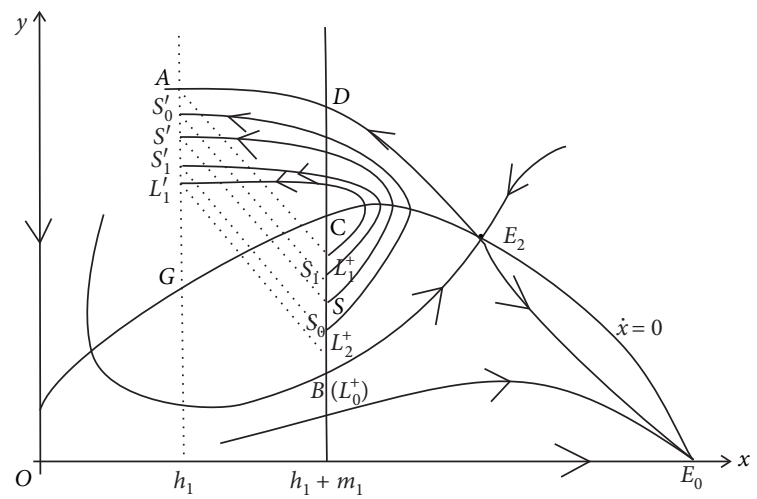

Figure 4: Existence of attraction region of system (11).

Repeating the above steps, two point sequences $\left\{L_{2 k}^{+}\right\}$and $\left\{L_{2 k+1}^{+}\right\}, k=0,1,2, \ldots$, separated by point $S$ are obtained, where sequence $\left\{y_{L_{2 k}^{+}}\right\} k=0,1,2, \ldots$ is monotonically increasing with $y_{L_{2 k}^{+}} \leq y_{S}$ while $\left\{y_{L_{2 k+1}^{+}}\right\}_{k=0,1,2, \ldots}$ is monotonically decreasing with $y_{S} \leq y_{L_{2 k+1}^{+}}$. We further suppose that

$$
\begin{gathered}
y_{L_{2 k}} \longrightarrow y_{S_{0}} \leq y_{S}, \quad \text { as } k \longrightarrow \infty \\
y_{L_{2 k+1}} \longrightarrow y_{S_{1}} \geq y_{S}, \quad \text { as } k \longrightarrow \infty
\end{gathered}
$$

And two points $S_{0}$ and point $S_{1}$ may coincide with each other, that is to say, points $S_{0}, S_{1}$, and $S$ are the same one.

Let the forward orbits $\pi_{+}\left(S_{0}\right), \pi_{+}\left(S_{1}\right)$ meet the impulsive set $x=h_{1}$ at points $S_{1}^{\prime}$ and $S_{1}^{\prime}$, respectively. Denote the region circled by the closed curve $\widehat{S_{0} S_{0}^{\prime}} \cup \overline{S_{0}^{\prime} S_{1}^{\prime}} \cup \widehat{S_{1}^{\prime} S_{1}} \cup \overline{S_{1} S_{0}}$ by $\triangle \Omega$, and we will prove that it is the attraction region of system (11) in $\widetilde{\Omega}$.

For any point $P_{0}^{+} \in \overline{B S_{0}}$, we can pick an integer $k>0$ such that $y_{L_{2 k}^{+}}<y_{P_{0}}<y_{L_{2(k+1)}^{+}}$. Then, similar to the above discussion, we can get a series of successor points $\left\{P_{2 i}^{+}\right\}$and $\left\{P_{2 i+1}^{+}\right\}$, $i=0,1,2, \ldots$, which satisfy that $\left\{y_{P_{2 i}^{+}}\right\}_{i=0,1,2, \ldots}$ is monotonically increasing with $y_{P_{2 i}^{+}} \leq y_{S_{0}}$ and $\left\{y_{P_{2 i+1}^{+}}\right\}_{i=0,1,2, \ldots}$ is monotonically decreasing with $y_{P_{2 i+1}^{+}} \geq y_{S_{1}}$. Besides, 


$$
\begin{aligned}
y_{P_{2 i+1}^{+}} & \longrightarrow y_{S_{1}}, \quad i \longrightarrow \infty \\
y_{P_{2 i}^{+}} & \longrightarrow g_{S_{0}}, \quad i \longrightarrow \infty
\end{aligned}
$$
$\triangle \Omega$.

Then, the orbit from $P_{0}$ eventually tends to the region

Furthermore, for any point $P_{0}^{+} \in \overline{S_{1} D}$, the forward orbit $\pi_{+}\left(P_{0}^{+}\right)$will intersect the phase set $x=h_{1}+m_{1}$ on $\overline{B S_{0}}$ after impulsive jumps once or twice and then is eventually attracted to the region $\triangle \Omega$. To sum up, we get that $\triangle \Omega$ is the attraction region of system (11) in $\widetilde{\Omega}$. This completes the proof.

Remark 3. If three points $S_{0}, S_{1}$, and $S$ coincide with each other, then the attraction region $\triangle \Omega$ is exactly the order-1 cycle $\Gamma_{S}$ we obtained in Theorem 3 . In this case, $\Gamma_{S}$ is globally orbitally asymptotically stable in $\widetilde{\Omega}$. Of course, these three points $S_{0}, S_{1}$, and $S$ may not coincide with each other; then, the attraction region $\triangle \Omega$ can be circled by an order- 2 orbit.

3.3. Analysis of System (12). For system (12), two impulsive sets can be defined as

$$
\begin{aligned}
& \mathscr{M}_{21}=\left\{(x, y) x=h_{1}, y>r h_{1}\left(1-\frac{h_{1}}{K}\right) e^{-\beta h_{1}}\right\}, \\
& \mathscr{M}_{22}=\left\{(x, y) x=h_{2}, y<r h_{2}\left(1-\frac{h_{2}}{K}\right) e^{-\beta h_{2}}\right\} .
\end{aligned}
$$

However, the corresponding phase sets are the same which is denoted by $\mathcal{N}_{2}=\left\{(x, y) \mid x=h_{1}+m_{1}=h_{2}-\right.$ $\left.m_{2} \triangleq n, y>0\right\}$.

For the convenience of the following discussion, let the unstable flow of $E_{2}\left(x_{2}, y_{2}\right)$ meet the straight lines $x=h_{1}, x=n$, and $x=h_{2}$ for the first time at points $A, D$, and $E$, while the stable flow of $E_{2}\left(x_{2}, y_{2}\right)$ meet the three lines for the last time at points $H, B$, and $K$ (in the direction of time increasing). Besides, the three lines intersect the isoclinic line $\dot{x}=0$ at points $G, C$, and $F$. For the forward orbit $\pi_{+}(F)$ and backward orbit $\pi_{-}(F)$, we denote their intersection points with $x=h_{1}$ and $x=n$ by $N$ and $Q$, respectively (see Figure 5 ).

Theorem 5. If $y_{Q}<(1-p) y_{N}<y_{C}, \quad \alpha \mu>e \beta D, \quad x_{2}<K<$ $(1 / \beta)+x_{1}, r(\beta-(1 / K))+\alpha \mu<2 \sqrt{((\beta r D) / K)}$, and $x_{1}<h_{1}<$ $n<h_{2}<x_{2}$, then system (12) admits an order-1 periodic orbit in the region $\Omega$.

Proof. Consider the point $L \in \mathcal{N}_{2}$. If $y_{L}<y_{Q}$, then the forward orbit $\pi_{+}(L)$ will firstly meet the second impulsive set $\mathscr{M}_{22}$ and goes through the corresponding impulsive jump, while if $y_{L}>y_{Q}$, then the forward orbit $\pi_{+}(L)$ will meet the first impulsive set $\mathscr{M}_{21}$ first and goes through the corresponding impulsive jump.

Similar to the analysis in Theorem 3, we can get the existence of the order-1 periodic solution $\Gamma_{S}$ for system (12), where $S \in \mathcal{N}_{2}$ satisfies $y_{Q}<y_{S}<y_{C}$. The proof is completed.

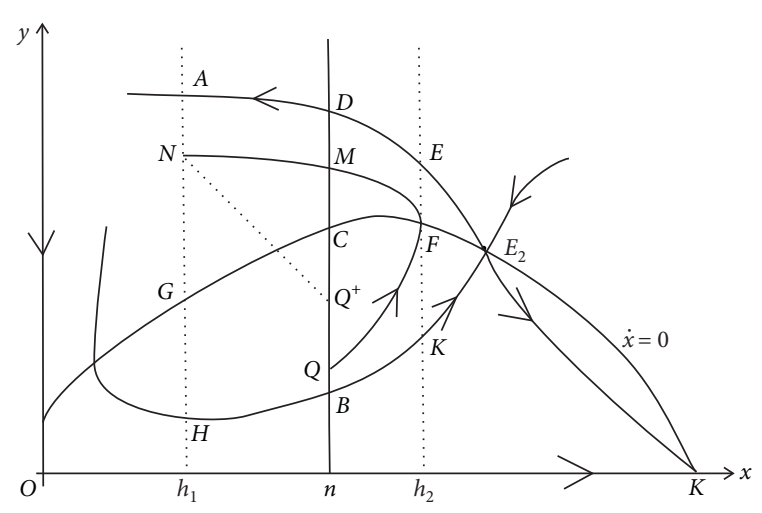

Figure 5: Illustration of the dynamics of system (12).

Remark 4. Similar to the discussion in Theorem 4, we can obtain that if $(1-p) y_{G}>y_{Q}$, then system (12) has a local attraction region which is circled by the closed curve $\overline{A G} \cup \widehat{G C} \cup \overline{C Q} \cup \widehat{Q F} \cup \overline{F E} \cup \widehat{E D A}$.

Theorem 6. If $\left(r(1-(n / K)) / \alpha e^{-\beta n}\right)<\tau<y_{D}-y_{F}$, $(1-p)$ $y_{A}<y_{Q}$, and $x_{1}<h_{1}<n<h_{2}<x_{2}$, then system (12) admits an order- 2 periodic orbit in $\Omega$ provided that conditions in Theorems 1 and 2 are satisfied.

Proof. Since $\tau>\left(r(1-(n / K)) / \alpha e^{-\beta n}\right)$, we have $y_{D}-y_{F}>\tau>$ $y_{C}$. Choose a point $A_{1} \in \mathcal{N}_{2}$ next to $Q$ satisfying $y_{A_{1}}<y_{Q}$, and let the forward orbit $\pi_{+}\left(A_{1}\right)$ meet the impulsive set $\mathscr{M}_{22}$ at point $A_{1}^{\prime}$ with $y_{A_{1}^{\prime}}<y_{F}$; then, $A_{1}^{\prime}$ is mapped to $B_{1} \in \mathcal{N}_{2}$. There is $y_{C}<y_{B}<y_{D}$ for $y_{D}-y_{F}>\tau>y_{C}$. The forward orbit $\pi_{+}\left(B_{1}\right)$ meets the impulsive set $\mathscr{M}_{21}$ at point $C_{1}$ with $y_{N}<y_{C_{1}}<y_{A}$; then, $C_{1}$ is mapped to $D_{1} \in \mathcal{N}_{2}$. Obviously, we have $y_{D_{1}}<y_{A_{1}}$. Then, $f_{\text {sor }}^{2}\left(A_{1}\right)=y_{D_{1}}-y_{A_{1}}<0$ (see the left figure in Figure 6).

Choose another point $A_{2} \in \mathcal{N}_{2}$ satisfying $0<y_{A_{2}} \ll 1$, and let the forward orbit $\pi_{+}\left(A_{2}\right)$ meet the impulsive set $\mathscr{M}_{22}$ at point $A_{2}^{\prime}$ with $y_{A_{2}^{\prime}}<y_{K}$; then, $A_{2}^{\prime}$ is mapped to $B_{2} \in \mathcal{N}_{2}$. There is $y_{C}<y_{B_{2}}<y_{B_{1}}$ for $y_{D}-y_{F}>\tau>y_{C}$. The forward orbit $\pi_{+}\left(B_{2}\right)$ meets the impulsive set $\mathscr{M}_{21}$ at point $C_{2}$ with $y_{G}<y_{C_{2}}<y_{C_{1}}$; then, $C_{2}$ is mapped to $D_{2} \in \mathcal{N}_{2}$. Obviously, $y_{D_{2}}>y_{A_{2}}$. Then, $f_{\text {sor }}^{2}\left(A_{2}\right)=y_{D_{3}}-y_{A_{2}}>0$.

Due to the continuity of $f_{\text {sor }}^{2}$, there must exist a point $S^{+} \in \overline{A_{1} A_{2}}$ such that $f_{\text {sor }}^{2}(S)=0$. Then, system (12) admits an order-2 periodic orbit. The proof is completed.

Remark 5. According to the proof of Theorem 5, system (12) may have order- $k(k>2)$ periodic orbits provided that the parameters satisfy certain conditions.

In the following, we discuss the stability of the order-2 periodic orbit $\widetilde{\Gamma}_{S}$ for system (12). Assume $\widetilde{\Gamma}_{S}$ passes through four points $S^{+}\left(n,(1-p) \eta_{0}\right), S\left(h_{1}, \eta_{0}\right), W\left(h_{2}, \zeta_{0}\right)$, and $W^{+}\left(n, \zeta_{0}\right.$ $+\tau$ ) (see the right figure in Figure 6) and its period is $T$.

Since the analytical expression of this order- 2 periodic solution cannot be obtained, we study its stability by using the criterion in Lemma 2. According to Lemma 2, we have 


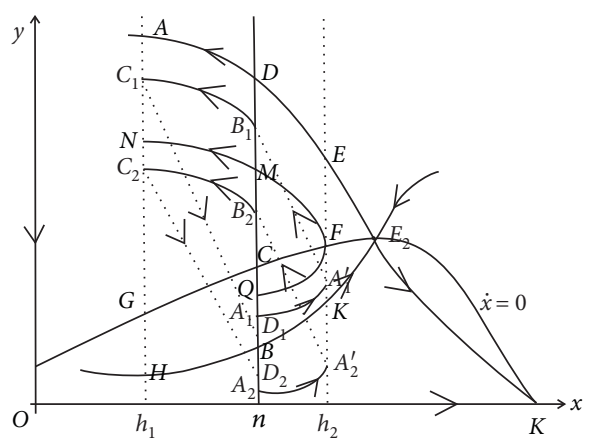

(a)

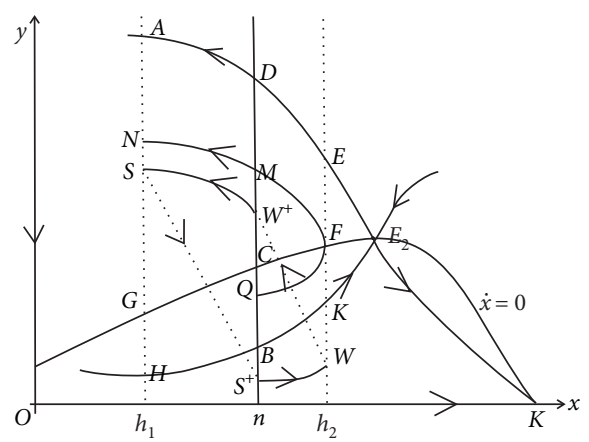

(b)

FIGURE 6: Existence of order-2 periodic orbit of system (12).

$$
\begin{gathered}
P(x, y)=r x\left(1-\frac{x}{K}\right)-\alpha x y e^{-\beta x}, \quad Q(x, y)=\mu \alpha x y e^{-\beta x}-D y, \\
\alpha_{1}(x, y)=m_{1}, \quad \beta_{1}(x, y)=-p y, \quad \varphi_{1}(x, y)=x-h_{1}, \\
\alpha_{2}(x, y)=-m_{2}, \quad \beta_{2}(x, y)=\tau, \quad \varphi_{2}(x, y)=x-h_{2} .
\end{gathered}
$$

Then, through simple calculation, we have

$$
\begin{aligned}
& \frac{\partial P}{\partial x}=r-\frac{2 r}{K} x-\alpha y(1-\beta x) e^{-\beta x}, \\
& \frac{\partial Q}{\partial y}=\alpha \mu x e^{-\beta x}-D, \\
& \frac{\partial \alpha_{1}}{\partial x}=\frac{\partial \alpha_{1}}{\partial y}=0, \\
& \frac{\partial \beta_{1}}{\partial x}=0, \\
& \frac{\partial \beta_{1}}{\partial y}=-p \\
& \frac{\partial \varphi_{1}}{\partial x}=1 \\
& \frac{\partial \varphi_{1}}{\partial y}=0 \\
& \frac{\partial \alpha_{2}}{\partial x}=\frac{\partial \alpha_{2}}{\partial y}=0 \\
& \frac{\partial \beta_{2}}{\partial x}=\frac{\partial \beta_{2}}{\partial y}=0, \\
& \frac{\partial \varphi_{2}}{\partial x}=1 \\
& \frac{\partial \varphi_{2}}{\partial y}=0
\end{aligned}
$$

and then

$$
\begin{aligned}
\Delta_{1} & =\frac{P_{+}\left(\left(\partial \beta_{1} / \partial y\right)\left(\partial \varphi_{1} / \partial x\right)-\left(\partial \beta_{1} / \partial x\right)\left(\partial \varphi_{1} / \partial y\right)+\left(\partial \varphi_{1} / \partial x\right)\right)+Q_{+}\left(\left(\partial \alpha_{1} / \partial x\right)\left(\partial \varphi_{1} / \partial y\right)-\left(\partial \alpha_{1} / \partial y\right)\left(\partial \varphi_{1} / \partial x\right)+\left(\partial \varphi_{1} / \partial y\right)\right)}{P\left(\partial \varphi_{1} / \partial x\right)+Q\left(\partial \varphi_{1} / \partial y\right)} \\
& =\frac{(1-p) n\left[r(1-(n / K))-\alpha(1-p) \eta_{0} e^{-\beta n}\right]}{h_{1}\left[r\left(1-\left(h_{1} / K\right)\right)-\alpha \eta_{0} e^{-\beta h_{1}}\right]}, \\
\Delta_{2} & =\frac{P_{+}\left(\left(\partial \beta_{2} / \partial y\right)\left(\partial \varphi_{2} / \partial x\right)-\left(\partial \beta_{2} / \partial x\right)\left(\partial \varphi_{2} / \partial y\right)+\left(\partial \varphi_{2} / \partial x\right)\right)+Q_{+}\left(\left(\partial \alpha_{2} / \partial x\right)\left(\partial \varphi_{2} / \partial y\right)-\left(\partial \alpha_{2} / \partial y\right)\left(\partial \varphi_{2} / \partial x\right)+\left(\partial \varphi_{2} / \partial y\right)\right)}{P\left(\partial \varphi_{2} / \partial x\right)+Q\left(\partial \varphi_{2} / \partial y\right)} \\
& =\frac{n\left[r(1-(n / K))-\alpha\left(\zeta_{0}+\tau\right) e^{-\beta n}\right]}{h_{2}\left[r\left(1-\left(h_{2} / K\right)\right)-\alpha \zeta_{0} e^{-\beta h_{2}}\right]} .
\end{aligned}
$$

Setting $\quad \mathfrak{H}(t)=(\partial P / \partial x)(\xi(t), \eta(t))+(\partial Q / \partial y)(\xi(t)$, $\eta(t))$, we can get

$$
\begin{aligned}
\tilde{\mu} & =\Delta_{1} \Delta_{2} \int_{0}^{T} \mathfrak{H}(t) \mathrm{d} t \\
& =\frac{(1-p) n^{2}\left[r(1-(n / K))-\alpha(1-p) \eta_{0} e^{-\beta n}\right]\left[r(1-(n / K))-\alpha\left(\zeta_{0}+\tau\right) e^{-\beta n}\right]}{h_{1} h_{2}\left[r\left(1-\left(h_{1} / K\right)\right)-\alpha \eta_{0} e^{-\beta h_{1}}\right]\left[r\left(1-\left(h_{2} / K\right)\right)-\alpha \zeta_{0} e^{-\beta h_{2}}\right]} \int_{0}^{T} \mathfrak{H}(t) \mathrm{d} t .
\end{aligned}
$$




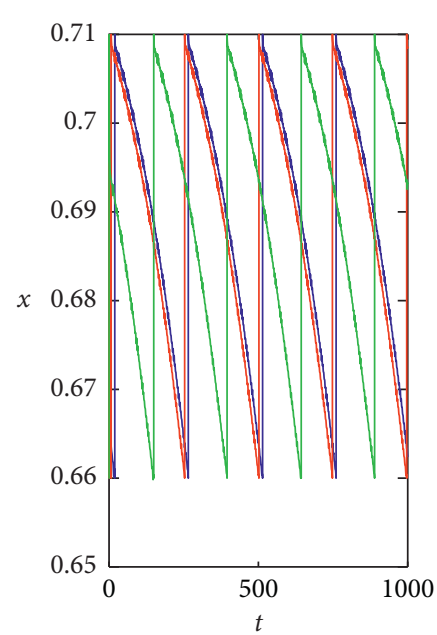

(a)

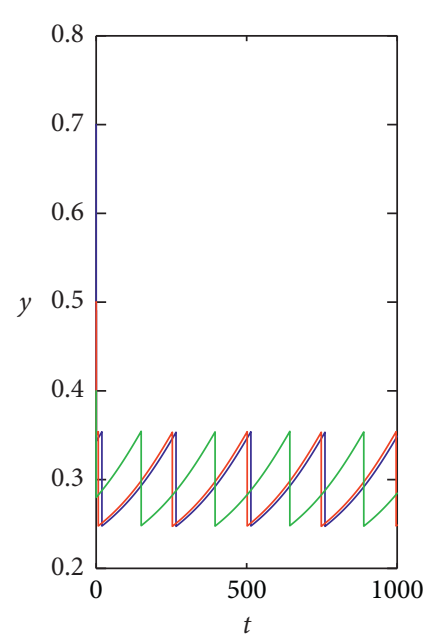

(b)

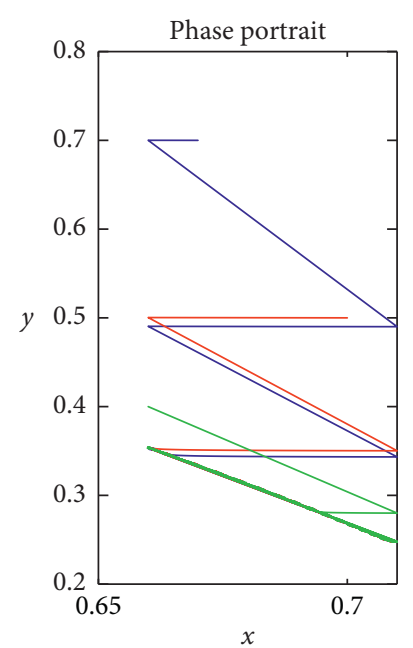

(c)

Figure 7: The order-1 periodic solution of system (11) with $p=0.3$.

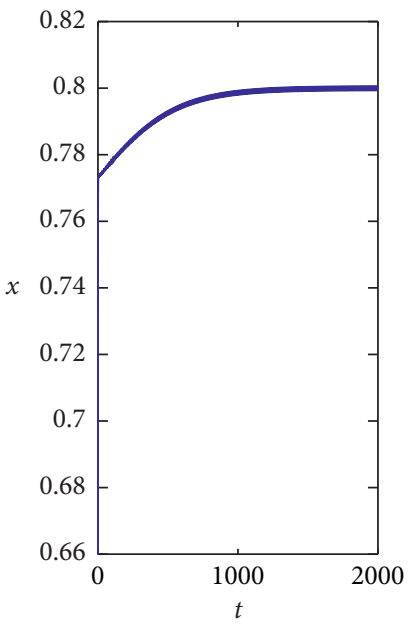

(a)

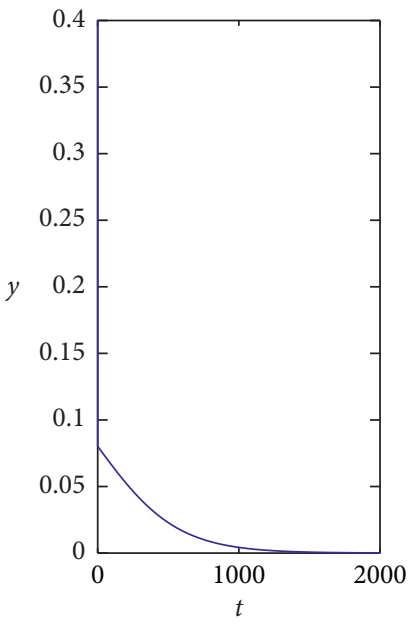

(b)

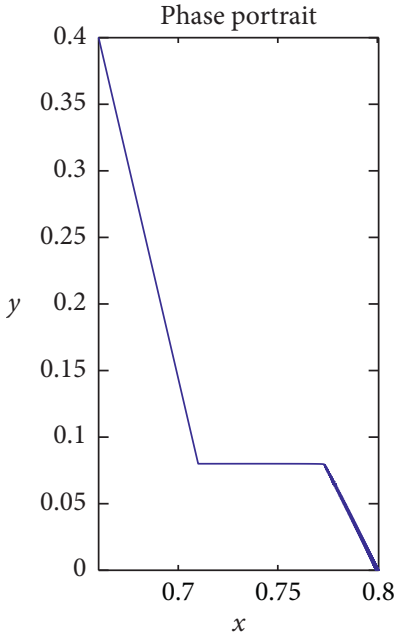

(c)

Figure 8: The predator species tends to be extinct for system (11) with $p=0.8$.

Then, we have the following result:

Theorem 7. If $\left(r(1-(n / K)) / \alpha e^{-\beta n}\right)<\tau<y_{D}-y_{F},(1-p)$ $y_{A}<y_{Q}$ and $x_{1}<h_{1}<n<h_{2}<x_{2}$, then the order-2 periodic solution of system (12) is stable provided that $|\widetilde{\mu}|<1$.

\section{Numerical Simulations}

In this section, a series of numerical simulations are carried out, which will on the one hand confirm the theoretical results and, on the other hand, reveal some peculiar dynamical phenomena.

4.1. Existence and Stability of Order-1 and Order-2 Periodic Orbit. To satisfy the conditions listed in Theorems 1 and 2, we choose the parameters as follows:

$$
\begin{aligned}
r & =1.5, \\
K & =0.8, \\
\alpha & =2, \\
\beta & =1.5, \\
\mu & =0.615, \\
D & =0.3 .
\end{aligned}
$$

Then, we can easily get $\alpha \mu=1.23>e \beta D \approx 1.223$, $r(\beta-(1 / K))+\alpha \mu=1.605<2 \sqrt{((\beta r D) / K)} \approx 1.8371, \quad x_{1} \approx$ $0.5995, x_{2} \approx 0.7395$, and $x_{2}<K<(1 / \beta)+x_{1}$. According to Theorems 1 and 2, system (1) admits four equilibria: two boundary equilibria $O(0,0)$ and $E_{0}(K, 0)$ and two positive steady states $E_{1}\left(x_{1}, y_{1}\right)$ and $E_{2}\left(x_{2}, y_{2}\right)$, where $O(0,0)$ and 


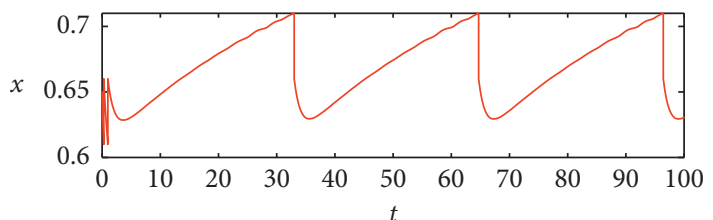

(a)

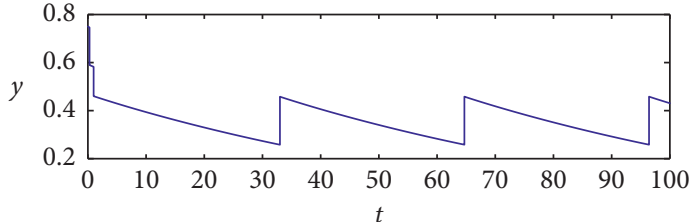

(b)

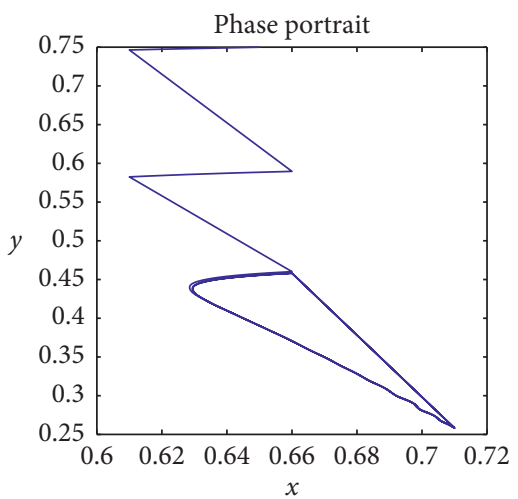

(c)

Figure 9: The order-1 periodic orbit of system (12). Control parameters: $p=0.7$ and $\tau=0.2$.

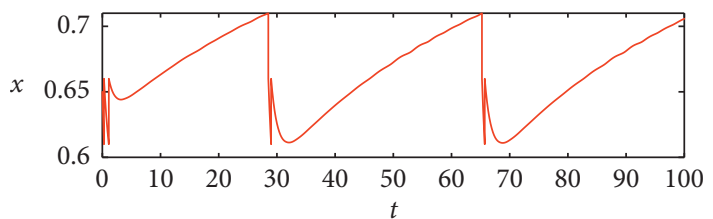

(a)

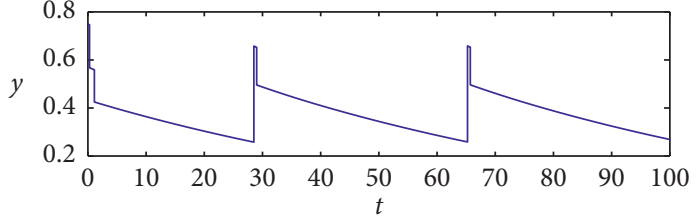

(b)

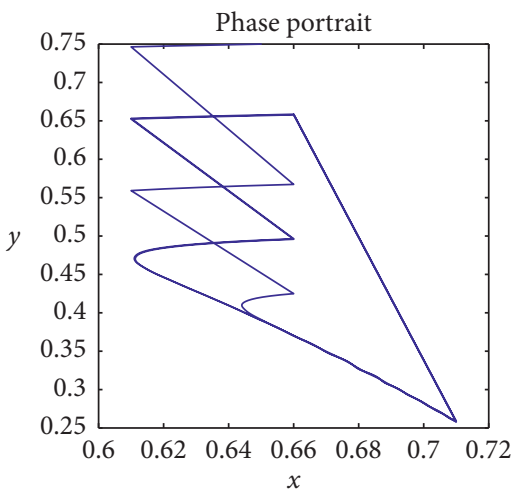

(c)

Figure 10: The order-2 periodic orbit of system (12). Control parameters: $p=0.6$ and $\tau=0.4$.

$E_{2}\left(x_{2}, y_{2}\right)$ are unstable saddle points while $E_{0}(K, 0)$ and $E_{1}\left(x_{1}\right.$, $\left.y_{1}\right)$ are stable ones.

For unilateral control system (11), we choose the lower control threshold $h_{1}=0.66>x_{1}$ and $m_{1}=0.05$, then there are a fixed parameter values $p^{*}$ such that system (11) admits an order-1 periodic orbit for some $p<p^{*}$ (see Figure 7), while it has no periodic orbit if $p>p^{*}$ (see Figure 8 ). Obviously, the order-1 periodic orbit in Figure 7 is orbitally stable.

For bilateral control system (12), we choose the two control thresholds $h_{1}=0.61>x_{1}$ and $h_{2}=0.71<x_{2}$, while $m_{1}$
$=0.05$ and $m_{2}=0.05$ such that the shared phase set is $x=n=$ 0.66. By changing the impulse parameter, we show that system (12) can have different types of periodic solution (see Figures 9-12).

When the order-2 periodic orbit of system (12) exists, we investigate its stability. Choose different initial values; we can see that this periodic solution is orbitally stable (see Figure 13).

4.2. Bifurcation Diagram of System (12). According to the discussion in Section 4.1, when we fix $p=0.6$, system (12) can 


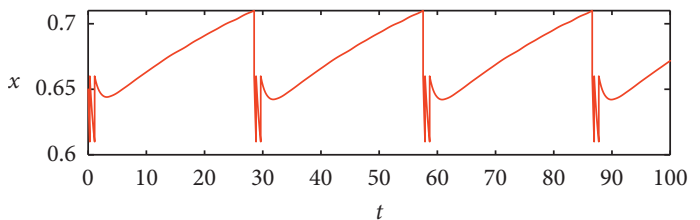

(a)

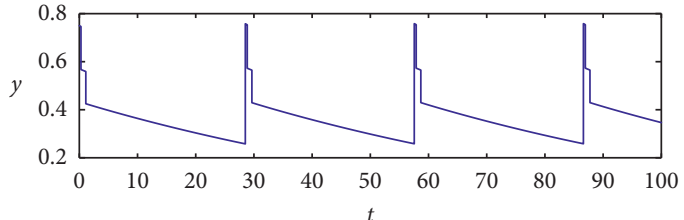

(b)

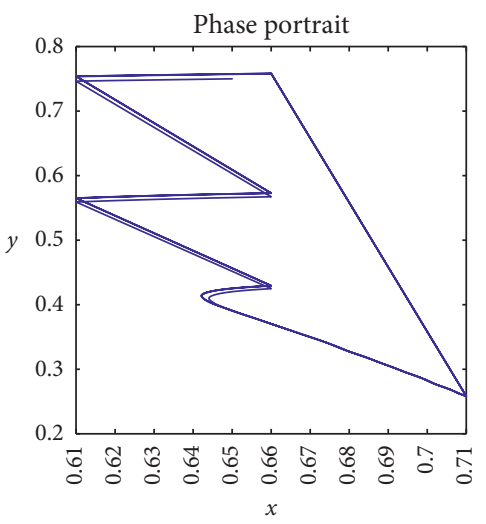

(c)

Figure 11: The order-3 periodic orbit of system (12). Control parameters: $p=0.6$ and $\tau=0.5$.

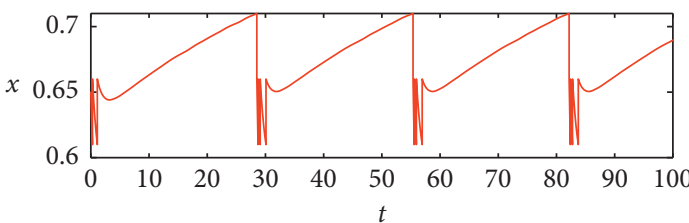

(a)

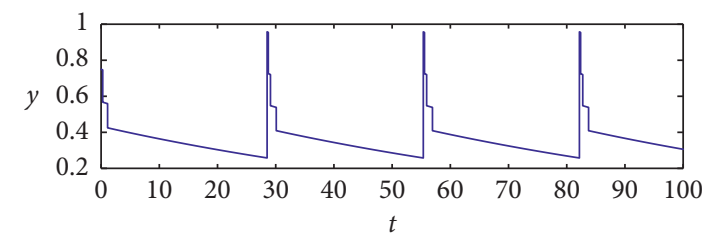

(b)

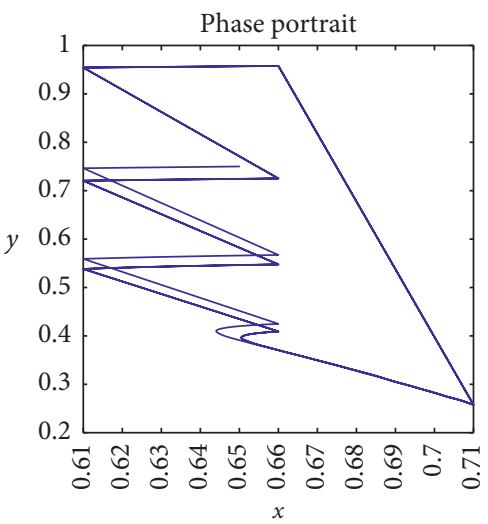

(c)

FIgURE 12: The order-4 periodic orbit of system (12). Control parameters: $p=0.6$ and $\tau=0.7$.

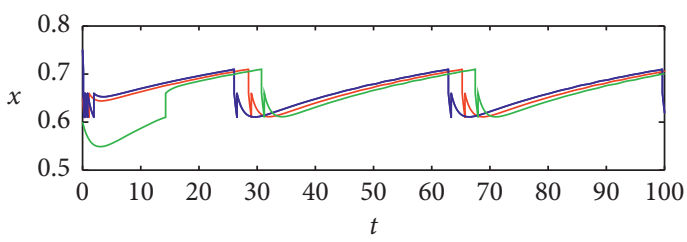

(a)

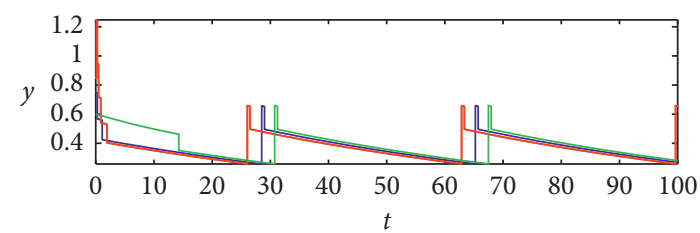

(b)

Figure 13: Continued. 


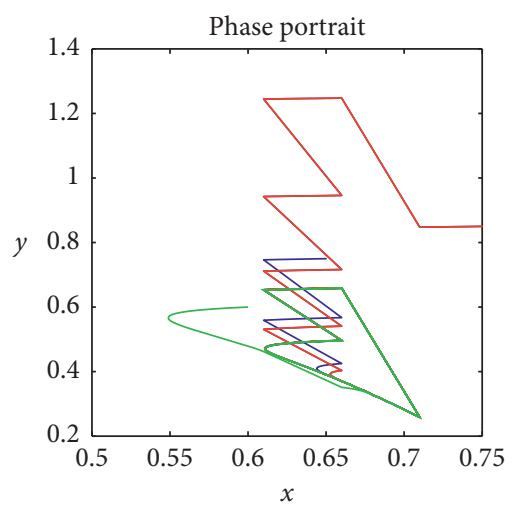

(c)

FIgURE 13: Orbital stability of the order-2 periodic solution of system (12). Control parameters: $p=0.6$ and $\tau=0.4$.

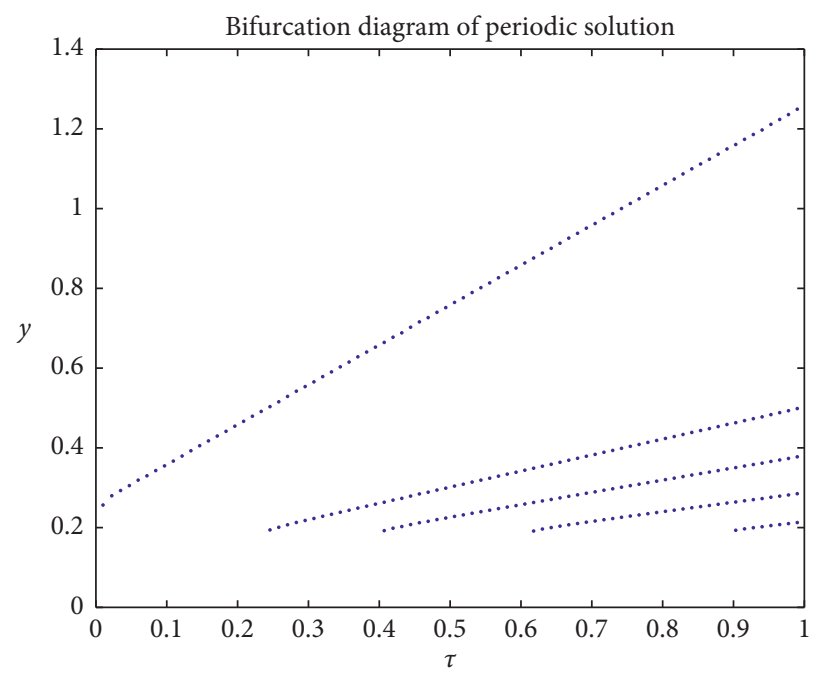

Figure 14: Bifurcation diagram of system (12) with respect to $\tau$ for $\tau \in[0,1]$.

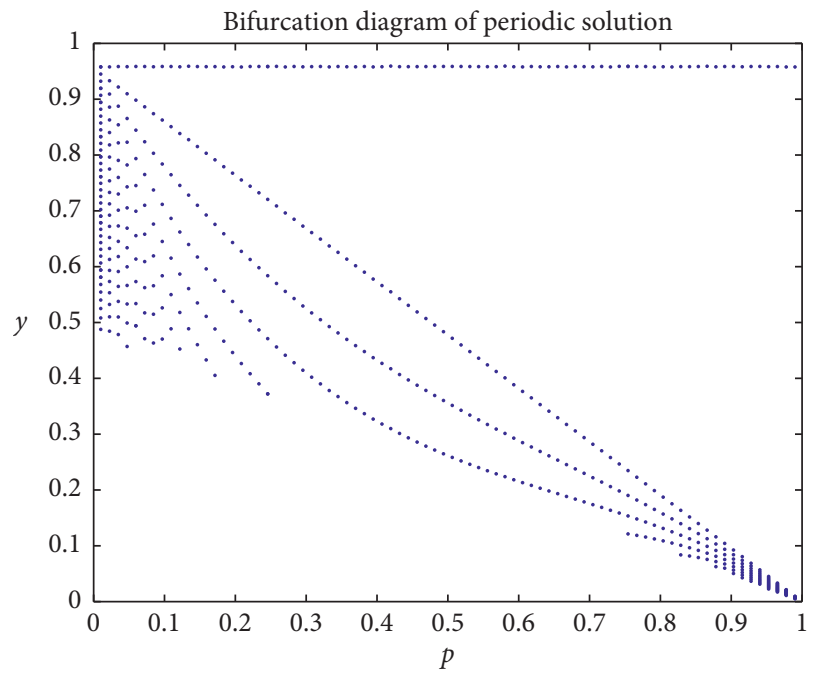

FIgURE 15: Bifurcation diagram of system (12) with respect to $p$ for $p \in(0,1)$. 

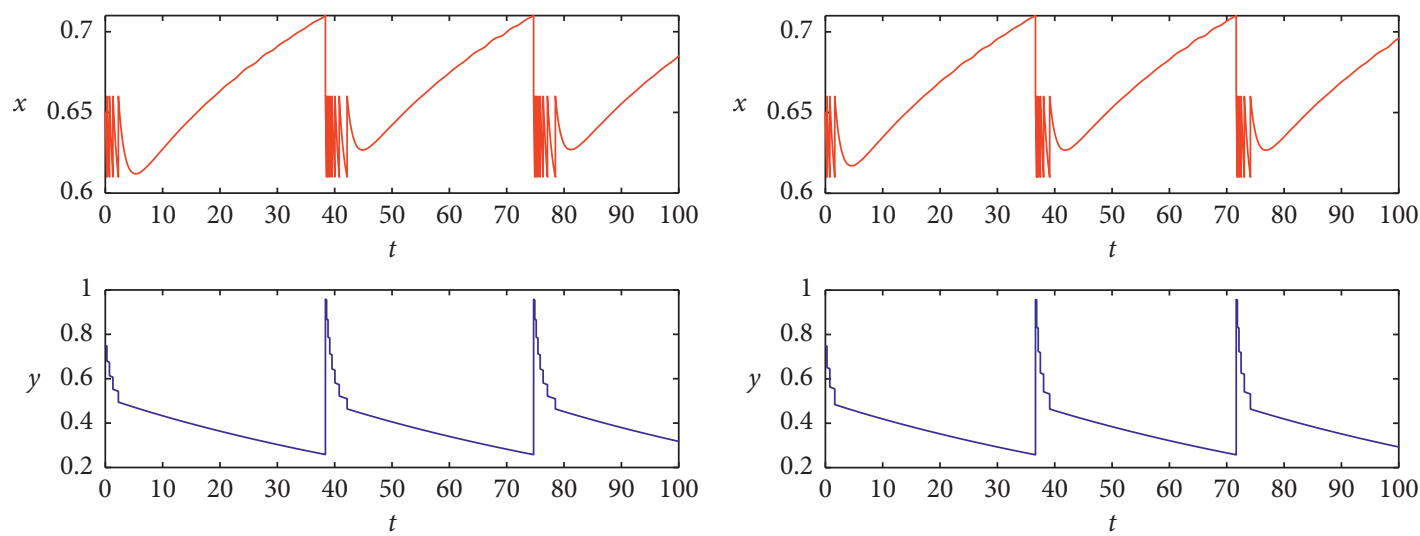

(a)

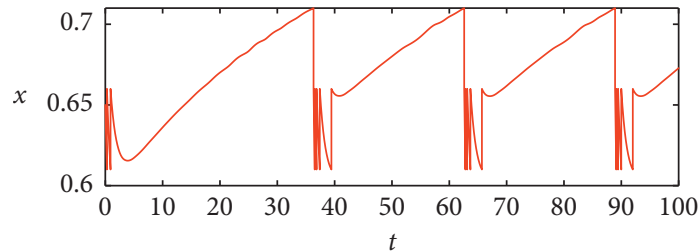

(b)

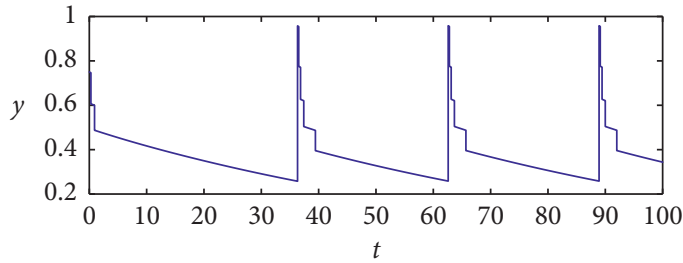

(c)
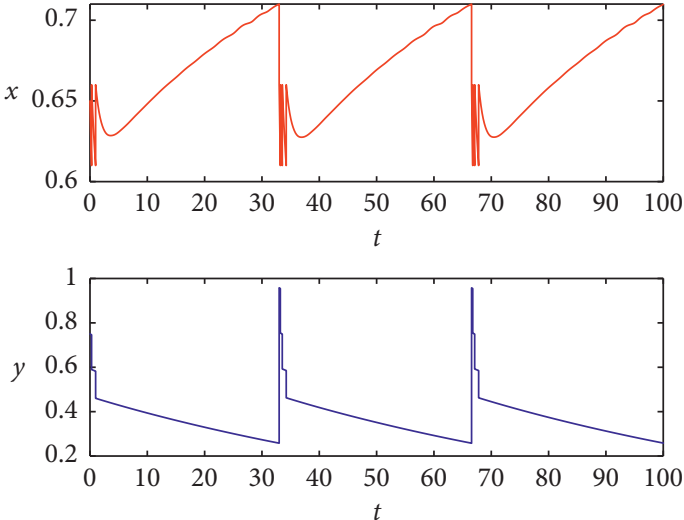

(d)

FiguRE 16: Change of the order of periodic orbits of system (12) for $p \in[0,0.3]$ : (a) order-8 periodic orbit at $p=0.1$; (b) order-6 periodic orbit at $p=0.15$; (c) order-5 periodic orbit at $p=0.25$; (d) order-4 periodic orbit at $p=0.3$.

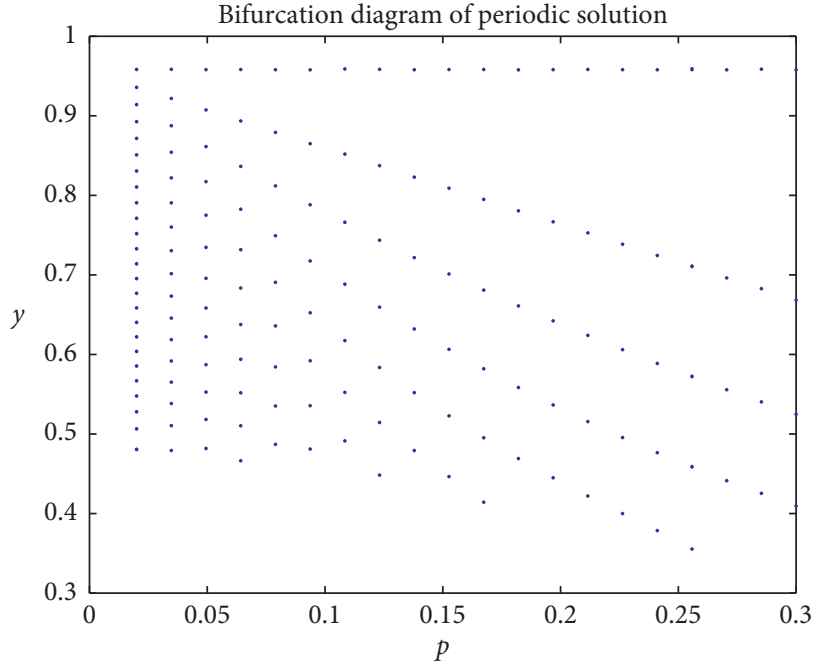

(a)

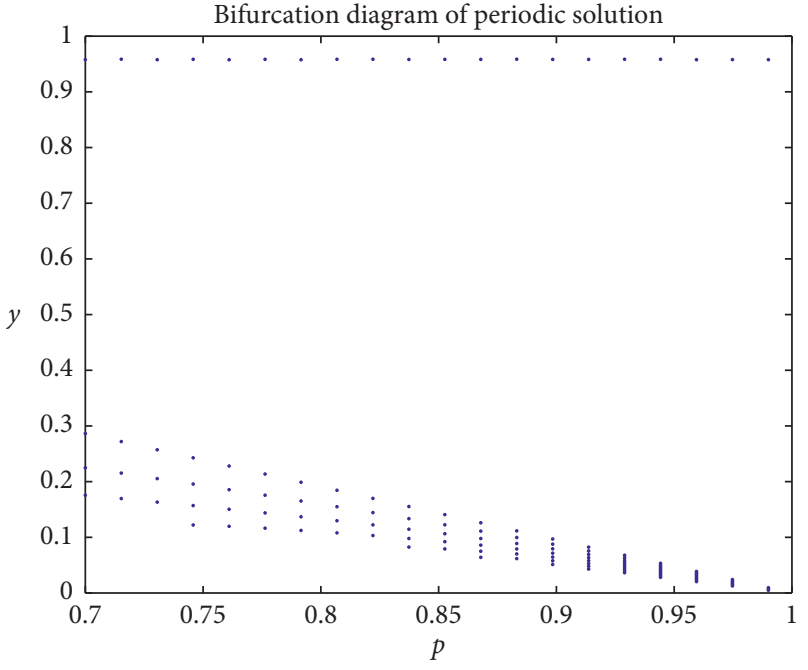

(b)

Figure 17: Bifurcation diagrams of system (12) with respect to $p$ for $p \in(0,0.3]$ and $p \in[0.7,1)$.

exhibit different types of periodic solution with the change of parameter $\tau$. We now exploit the bifurcation phenomenon with respect to $\tau$. For this end, we first set $p=0.6$, and
Figure 14 shows the bifurcation diagram of system (12) with respect to $\tau(\tau \in[0,1])$. From Figure 14, we find that system (12) can exhibit several types of periodic solution, such as 

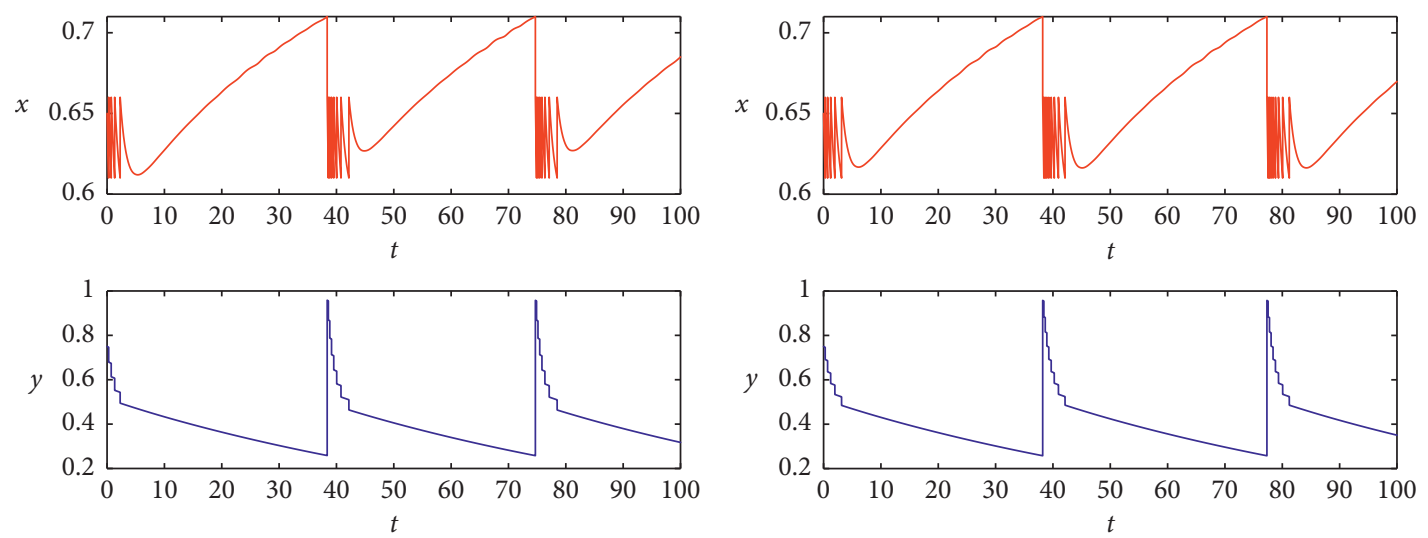

(a)

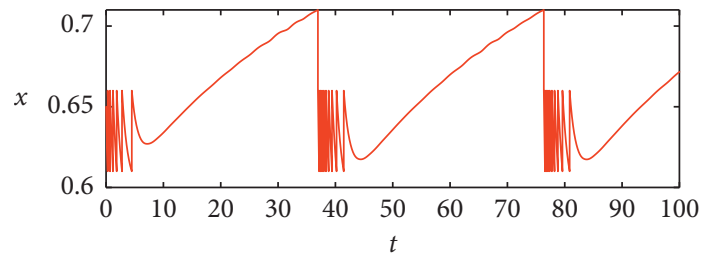

(b)

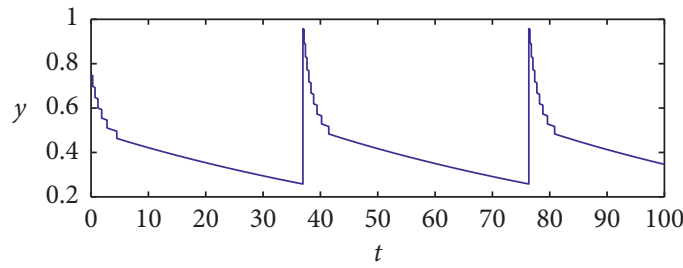

(c)
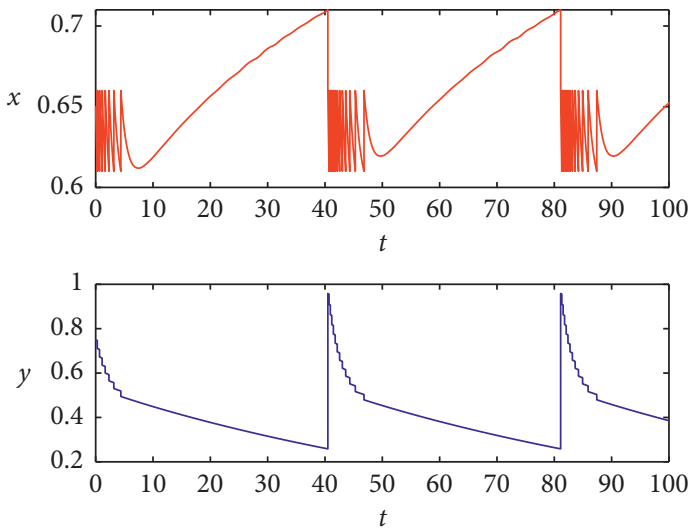

(d)

FiguRe 18: Change of the order of periodic orbits of system (12) for $p \in[0.9,0.95]$ : (a) order-8 periodic orbit at $p=0.9$; (b) order-9 periodic orbit at $p=0.92$; (c) order-10 periodic orbit at $p=0.93$; (d) order-13 periodic orbit at $p=0.95$.

order- $k$ periodic solution, $k=1,2,3,4,5$. However, the order bifurcation cannot lead to chaos. This is somewhat similar to a discrete dynamical system.

Besides, from Figure 14, we can see that orders 1, 2, 3, 4, and 5 periodic orbits exist for wide ranges of $\tau$. With the increase of $\tau$, there is an order- 1 periodic orbit when $\tau \in[0$, 0.2401 ) and then a positive order- 2 periodic solution bifurcates from the order- 1 periodic solution at $\tau \approx 0.2401$ through a fold bifurcation. The order- 2 periodic orbit exists when $\tau \in(0.2401,0.4031)$; then, a positive order-3 periodic solution bifurcates from the order-2 periodic solution at $\tau \approx$ 0.4031 . Analogously, a positive order-4 periodic solution bifurcates from the order- 3 periodic solution at $\tau \approx 0.6165$ and a positive order- 5 periodic solution bifurcates from the order-4 periodic solution at $\tau \approx 0.8952$.

In addition, we also set $\tau=0.7$ and observe the bifurcation phenomenon with respect to $p$. Figure 15 shows the bifurcation diagram of system (12) with respect to $p(p \in$ $(0,1))$. Similarly, we find that system (12) also exhibits many types of periodic solution, and the order bifurcation does not lead to chaos. Furthermore, for wide ranges of $p$, system (12) has periodic solutions of order 4 (for example $p$ $\epsilon(0.3,0.7)$ (see case $p=0.6$ in Figure 12) or order 5 (for example, $p \in(0.18,0.25] \cup(0.75,0.82)$, see case $p=0.25$ in Figure 16(c)).
Besides, from Figure 15, we can see that, with the change of $\tau$, there are two small subintervals in which the order of the periodic solution rises and falls dramatically. Figures in Figure 17 are two zoomed parts in the parameter interval $p \in(0$, $0.3]$ and $p \in[0.7,1)$. From the left one, we can see that the order of the periodic solution is very high when $p$ is near zero and then gradually drops down to 4 (for example, $0.28<p<0.3$ ). However, from the right one, we can see that the order of the periodic solution rises from $4(0.7<\tau<0.73)$ to a high level.

The time series of system (12) are present in Figure 16 for four different values of $p \in(0,0.3]$. We can see easily that the order of the periodic solution changes greatly. Similarly, the time series of the system (12) for $p \in[0.9,0.95]$ are present in Figure 18 and the order also changes dramatically. Obviously, these profiles of system (12) are associated with Figures 15 and 17.

\section{Conclusion}

In this paper, mathematical models for the management of two kinds of biological resources based on a given predatorprey relationship are proposed, and two types of control strategies, unilateral and bilateral control with the state feedback of the prey population, are investigated both theoretically and numerically. 
For the basic predator-prey system (1), we extend and renew the study of [26] and find conditions under which system (1) has two positive equilibria while $E_{1}\left(x_{1}, y_{1}\right)$ is a stable saddle and $E_{2}\left(x_{2}, y_{2}\right)$ is an unstable saddle (Theorem 1). Besides, we also provide conditions that can guarantee that the system (refer to system (1)) has no closed orbits (refer to Theorem 2).

Through analysis of the phase diagram, we find that the basic system has risk of resource depletion without control measures. Then, we introduce two types of control strategies, unilateral and bilateral control, both of which apply impulsive state feedback control technique. By applying the method of successor function, the existence of the order- 1 homoclinic orbit and order- 1 periodic orbit of the unilateral control system is obtained (refer to Theorem 3) and the attraction region of this system is also exploited (refer to Theorem 4). The phenomenon of homoclinic bifurcation is discussed by choosing $p$ as the bifurcation parameter. We first verify the existence of the bifurcation point $p^{*}$ and then show that if $p=p^{*}$, system (11) admits an order-1 homoclinic cycle; when parameter $p$ gradually decreases from $p=p^{*}$ to $p<p^{*}$, the homoclinic cycle disappears while an order-1 periodic orbit appears; when parameter $p$ gradually increases from $p=$ $p^{*}$ to $p^{*}<p<1$, the homoclinic cycle disappears but no new order-1 periodic cycle appears. Besides, by using geometry theory and Analogue of Poincaré criterion, we investigate the existence and stability of positive periodic solutions of the bilateral control system (refer to Theorem 5-7). Furthermore, a series of numerical simulations (including bifurcation diagram of periodic solution) are performed which not only confirm the theoretical results but also reveal some peculiar dynamical phenomena, such as the appearance of high-order periodic solutions and existence of parameter intervals with drastic order change of periodic solution.

Our study show that both unilateral control and bilateral control are beneficial to the biodiversity of the ecological system we studied in this paper. They can reduce the risk of resource depletion to some extent. From the unilateral control, we can see that the risk of extinction of the predator population still exists. That is, when the density of the predator is low enough while the prey population continues to increase, the predator will go extinct. However, the bilateral control can avoid the extinction of both species. Therefore, when comparing the two management strategies, our study encourages bilateral control rather than unilateral control for the risk of predator extinction.

\section{Data Availability}

The data used to support the findings of this study are included within the article.

\section{Conflicts of Interest}

The authors declare that they have no conflicts of interest.

\section{Acknowledgments}

This work was supported by the National Natural Science Foundation of China (11901502, 11671346, and 11871415), Nanhu Scholars Program of XYNU, Nanhu Scholars Program for Young Scholars of XYNU, and Foundation for Distinguished Young Talents in Higher Education of Henan (2019GGJS157).

\section{References}

[1] P. D. N. Srinivasu and S. D. Zawka, "Renewable resource management in a seasonally fluctuating environment with restricted harvesting effort," Mathematical Biosciences, vol. 301, pp. 1-9, 2018.

[2] C. W. Clark, Mathematical Bioeconomics: The Optimal Management of Renewable Recourses, Wiley, New York, NY, USA, 2nd edition, 1990.

[3] K. K. Tapan and M. Swarnakamal, "Optimal control of a fishery under critical depensation," Journal of Fisheries and Aquatic Science, vol. 1, no. 3, pp. 253-261, 2006.

[4] J. N. Sanchirico, U. Malvadkar, A. Hastings, and J. E. Wilen, "When are No-take zones an economically optimal fishery management strategy?" Ecological Applications, vol. 16, no. 5, pp. 1643-1659, 2006.

[5] M. Jerry and N. Raïssi, "The optimal strategy for a bioeconomical model of a harvesting renewable resource problem," Mathematical and Computer Modelling, vol. 36, no. 11-13, pp. 1293-1306, 2002.

[6] R. Hilborn, C. J. Walters, and D. Ludwig, "Sustainable exploitation of renewable resources," Annual Review of Ecology and Systematics, vol. 26, no. 1, pp. 45-67, 1995.

[7] H. R. Clarke and R. M. Shrestha, "Management of a renewable resource with a backstop substitute and nonautonomous prices," Journal of Environmental Economics and Management, vol. 14, no. 2, pp. 159-182, 1987.

[8] A. T. Charles and G. R. Munro, "Irreversible investment and optimal fisheries management: a stochastic analysis," Marine Resource Economics, vol. 1, no. 3, pp. 247-264, 1985.

[9] G. R. Munro, "The optimal management of transboundary renewable resources," The Canadian Journal of Economics, vol. 12, no. 3, pp. 355-376, 1979.

[10] Z. X. Lou, "Optimal control for a predator-prey system with age-dependent," International Journal of Biomathematics, vol. 2, no. 1, pp. 45-59, 2009.

[11] E. Lindkvist, O. Ekeberg, and J. Norberg, "Strategies for sustainable management of renewable resources during environmental change," Proceedings of the Royal Society B: Biological Sciences, vol. 284, no. 1850, Article ID 20162762, 2017.

[12] L. K. Sandal and S. I. Steinshamn, "A stochastic feedback model for optimal management of renewable resources," Natural Resource Modeling, vol. 10, no. 1, pp. 31-52, 1997.

[13] A. Leizarowitz and Y. Tsur, "Renewable resource management with stochastic recharge and environmental threats," Journal of Economic Dynamics and Control, vol. 36, no. 5, pp. 736753, 2012.

[14] J. M. Fuertes, "Optimal state feedback based resource allocation for resource-constrained control tasks," in Proceedings of the IEEE International Real-Time Systems Symposium, IEEE Computer Society, Lisbon, Portugal, December 2004.

[15] A. Leung and A.-Y. Wang, "Analysis of models for commercial fishing: mathematical and economical aspects," Econometrica, vol. 44, no. 2, pp. 295-303, 1976. 
[16] D. L. Ragozin and G. Brown, "Harvest policies and nonmarket valuation in a predator-prey system," Journal of Environmental Economics and Management, vol. 12, no. 2, pp. 155168, 1985.

[17] M. Mesterton-Gibbons, "On the optimal policy for combining harvesting of predator and prey," Natural Resource Modeling, vol. 3, no. 1, pp. 63-90, 1988.

[18] M. Fan and K. Wang, "Optimal harvesting policy for single population with periodic coefficients," Mathematical Biosciences, vol. 152, no. 2, pp. 165-178, 1998.

[19] L. K. Sandal and S. I. Steinshamn, "A feedback model for the optimal management of renewable natural capital stocks," Canadian Journal of Fisheries and Aquatic Sciences, vol. 54, no. 11, pp. 2475-2482, 1997.

[20] Q. Xiao and B. Dai, "Heteroclinic bifurcation for a general predator-prey model with Allee effect and state feedback impulsive control strategy," Mathematical Biosciences and Engineering, vol. 12, no. 5, pp. 1065-1081, 2015.

[21] M. Z. Huang, S. Z. Liu, X. Y. Song, and L. S. Chen, "Periodic solutions and homoclinic bifurcation of a predator-prey system with two types of harvesting," Nonlinear Dynamics, vol. 73, no. 1-2, pp. 815-826, 2013.

[22] Z. Q. Liang, G. P. Pang, and X. P. Zeng, "Qualitative analysis of a predator-prey system with mutual interference and impulsive state feedback control," Nonlinear Dynamics, vol. 87, no. 3, pp. 1-15, 2016.

[23] Y. Chen and Z. Zhao, "Mathematical model for continuous delayed single-species population with impulsive state feedback control," Journal of Applied Mathematics and Computing, vol. 61, no. 1-2, pp. 451-460, 2019.

[24] H. Guo, L. Chen, and X. Song, "Qualitative analysis of impulsive state feedback control to an algae-fish system with bistable property," Applied Mathematics and Computation, vol. 271, pp. 905-922, 2015.

[25] J.-B. Fu and L.-S. Chen, "Modelling and qualitative analysis of water hyacinth ecological system with two state-dependent impulse controls," Complexity, vol. 2018, Article ID 4543976, 16 pages, 2018.

[26] D. Xiao and S. Ruan, "Codimension two bifurcations in a predator-prey system with group defense," International Journal of Bifurcation and Chaos, vol. 11, no. 8, pp. 2123-2131, 2001.

[27] S. Li, Z. Xiong, and X. Wang, "The study of a predator-prey system with group defense and impulsive control strategy," Applied Mathematical Modelling, vol. 34, no. 9, pp. 25462561, 2010.

[28] Z. He, "Impulsive state feedback control of a predator-prey system with group defense," Nonlinear Dynamics, vol. 79, no. 4, pp. 2699-2714, 2015.

[29] P. S. Simenov and D. D. Bainov, "Orbital stability of the periodic solutions of autonomous systems with impulse effect," Publications of the Research Institute for Mathematical Sciences, vol. 25, no. 3, pp. 321-346, 1989.

[30] E. M. Bonotto and M. Federson, "Limit sets and the PoincaréBendixson theorem in impulsive semidynamical systems," Journal of Differential Equations, vol. 244, no. 9, pp. 23342349, 2008.

[31] L. S. Chen, "Pest control and geometric theory of semicontinuous dynamical systems," Beihua University Journal, vol. 12, no. 1, pp. 1-9, 2011. 